Panamerican Journal of

Thrauma, Critical Care \&e

Emergency

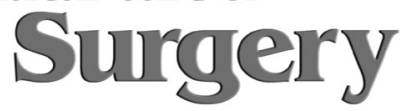

Revista Panamericana de

Thrauma, Cuidados Críticos

y Cirugía de Ainn ergerocia

Vol. 8 (Issue 2) May-August 2019 DOI: 10.5005/jp-journals-10030-1242

\title{
Trauma Abstracts
}

$32^{0}$ Panamerican Congress of Trauma, Critical Care and Emergency Surgery $92^{\circ}$ Congreso Chileno e Internacional de Cirurgía

Centro de Convención Enjoy Coquimbo Hotel de la Bahia and Hotel Club La Serena

November 13-16, 2019 
Avaliação Epidemiológica Das Vítimas Fatais Por Acidentes De Trânsito No Estado De Sergipe No Ano De 2017. Sonia O Lima, Maria J Nardelli, Yasmin S Melo, Renata LB De Andrade, Yasmim, Arthur V Déda, Edna S Dias, Adriana M Da Silva, Josilda F Cruz, Denison P Da Silva. From the Universidade Tiradentes, Aracaju/Se, Brazil.

Introducción: Acidentes de trânsito são a oitava maior causa de morte no planeta. Segundo o relatório da Organização Mundial de Saúde, a cada dia morrem no mundo, em acidentes de trânsito, mais de 3.000 pessoas, com a estimativa de uma morte a cada trinta segundos. Objetivo: Traçar o perfil demográfico e a análise espacial das vítimas fatais por acidentes de trânsito no estado de Sergipe. Materiales y métodos: Estudo ecológico, documental, realizado mediante avaliação dos laudos cadavéricos, atestados de óbito e dos boletins de ocorrência encaminhados pelas delegacias do estado ao Instituto Médico Legal de Sergipe, no período de janeiro a dezembro de 2017. Aprovado pelo CEP sob no 556.026. Resultados: Nesse período, 2.298 cadáveres deram entrada no Instituto Médico Legal de Sergipe, dos quais 392 foram por acidentes de transporte terrestre, o que representa $17 \%$. O gênero masculino foi o mais vitimado e o mais acometido. Os usuários ditos vulneráveis (pedestres, ciclistas e motociclistas) sofreram a maioria dos acidentes, sendo os condutores de ciclomotores e motocicletas o grupo que mais foi vitimado dentre todas as categorias (60,7\%). Quanto à faixa etária, $45,9 \%$ óbitos ocorreram em indivíduos entre 20 e 39 anos. Entre as vítimas de atropelamento destacam-se os idosos, que corresponderam a $35 \%$ destes. As vítimas não alfabetizadas ou que possuíam até o $5^{\circ}$ ano do ensino fundamental, representaram $43,6 \%$ da amostra total. A etnia parda foi maioria, representando $85,7 \%$ da amostra. Conclusiones:Oestado de Sergipe apresenta proporção ecaracterísticas das vítimas de mortes por acidentes fatais de trânsito semelhantes à literatura nacional e prevalência superior em acidentes envolvendo motociclistas. Indivíduos do gênero masculino, jovens, pardos, solteiros e com baixo grau de instrução foram os mais vitimados. Prevaleceram os acidentes aos domingos, no período noturno e na mesorregião do Leste Sergipano. O traumatismo cranioencefálico foi a principal causa mortis encontrada.

A Meta-analysis Assessing the Use of Anticoagulation and Antiplatelets After Vascular Trauma. Shujhat Khan, Hussein Elghezaly, Areeb Mian, Mansoor Khan. From the Imperial College London, London, UK.

Introduction: There is much debate regarding whether to use anticoagulation in vascular surgery following trauma because of the increased risk of local and systemic haemorrhage. Currently, during elective arterial repair, systemic anticoagulation is routinely provided during arterial reconstruction and peripheral artery. We aimed to systematically review the evidence of the impact of anti-platelets and anti-coagulants in the prognosis following vascular trauma. Materials and methods: A search was conducted for studies comparing anticoagulation and/or anti-platelet therapies compared to no treatment in the context of vascular trauma. The MEDLINE database was searched using Ovid for keywords and MeSH terms including "Anticoagulation", "Vascular Surgery", and "Wounds and Injuries". Results: A total of 604 citations were identified from the literature search. Eight clinical studies involving a total of 1807 patients met the inclusion criteria for this review whilst seven clinical studies involving a total of 1383 patients were included for the statistical analysis. Four studies were identified that demonstrated positive outcomes with the use of anticoagulants in the context of vascular trauma. Meta-analysis revealed a significant association between systemic anticoagulation use and reduction in negative outcomes (weighted $\mathrm{OR}=0.46 ; 95 \% \mathrm{Cl}$ $0.34-0.64 ; p<0.00001$. Patients in the anticoagulation arm had a lower amputation rate compared to those who were not given intraoperative anticoagulation (weighted OR 0.42; 95\% Cl 0.22-0.78; $p=0.007$ ). Additionally, patients in the anticoagulation arm had lower negative outcomes compared to those who were not given intraoperative coagulation (weighted OR $0.31 ; 95 \% \mathrm{Cl} 0.14-0.67 ; p=0.003$ ). Conclusion:This meta-analysis has demonstrated that use of systemic anticoagulants in the context of vascular trauma is significantly associated with a reduction in failure of arterial patency, amputation rates and VTE development. Thereby we cautiously recommend the use of anticoagulants in the general context of vascular trauma provided no contraindications exist.

Evaluation of Inhalation Injury: Is Higher Level of Care Always Beneficial? Christine Bierema, James Crodick, Michael Feldman. From the Virginia Commonwealth University, Virginia, USA.

Introduction: Not all patients with facial burns and/or smoke exposure require intubation or have significant inhalation injury. Burn practitioners must be able to differentiate between those who need a secure airway vs those who can be managed more conservatively. While the gold standard for diagnosing inhalation injury is bronchoscopic examination, it is not always available, is not therapeutic, and carries significant risk for the patient. The objective of our study was to determine if patients with mechanism of smoke exposure, but without signs and symptoms of smoke inhalation and without significant signs of injury on direct laryngoscopic examination, could safely be observed in a progressive care setting without escalation of care or elective intubation. Materials and methods:Weidentified patients who wereadmitted with diagnoses of inhalation injury or airway observation (with or without other cutaneous injuries) between January 1, 2014 and December 31, 2016. These patients were identified via CPT codes. Those patients' medical records were reviewed to determine if patients who were monitored but not intubated later required respiratory intervention. Results: Of the patients who met the inclusion criteria, no patients who were admitted for observation later required intubation for respiratory compromise. Conservative management through close airway observation was not associated with poor outcomes, but, in patients without cutaneous burns, was associated with a decrease in average ICU days (1.16 vs 3.18) and total average lengths of stay (1.72 vs 4.18). Conclusion: Over the three years, zero of the 29 patients who met criteria for observation required subsequent respiratory intervention. These patients presenting after smoke exposure can, in certain situations, be managed without using intensive care resources if they do not meet criteria for an emergent airway. Implementing this care process may help reduce ICU days, decreased overall lengths-of-stay, lower costs of care provision, and help improve staffing patterns.

Access to Emergent Surgical Care in Amazonas; Assessment of the Patient Journey. Gabriele Silva, Marinho, Emanoel N Costa, Carlos V De Almeida Luz, Jania A Ramos, Isabelle, Paul Truché, Lina Roa, José Emerson, Rodrigo V Ferreira. From the Universidade Do Estado Do Amazonas, Amazonas, Brazil. 
Introduction: To optimize surgical outcomes, the Lancet Commission on Global Surgery recommends access to essential surgical care within two hours. The state of Amazonas in Brazil has a large territory with unique hydrographic characteristics and a widely spread population resulting in barriers to receiving surgical care including seeking care, reaching care, and receiving care as outlined in the "three delay model."This study aims to assess access to surgical care in Amazonas based on the three-delay model. Materials and methods: This is a prospective, observational study of patients who underwent emergent abdominal surgery at Hospital and Pronto Socorro 28 de Agosto, a tertiary hospital in the city of Manaus, over a period of four weeks from July 2017-August 2017. A questionnaire was developed to assess patients' experience with each of the three delays to care. Hospital data was used to collection time to definitive surgical care among the patients. Results: 87 patients underwent emergent surgery. The overall median time to seek care 8 hours (IQR $=20.8) .28 \%$ of patients reached the hospital within two hours, with median travel time of 4.4 hours (IQR $=23.3$ ). Upon arrival, $79 \%$ of patients underwent surgery within 24 hours of arrival to the hospital, with the median time from arrival to surgery of 7 hours (IQR $=8.1$ ). Conclusion: The majority of patients in the state of Amazonas do not have timely access to essential emergent surgical care and experience all three delays of the "three delay model." There is a need to address the geographic, cultural and structural barriers in Amazonas in order to improve access to high quality surgical care.

Evolução Da Vítima Com Trauma Cranioencefálico Na Sala De Emergência. Lilia De Souza Nogueira, Hosana Da Silva, Regina MC De Sousa. From the Escola de Enfermagem da Universidade de São Paulo, São Paulo, Brazil.

Introducción: A frequência de vítimas de trauma cranioencefálico (TCE) nos serviços de emergência vem aumentando, e estudos que analisam a evolução dessas vítimas durante a permanência na sala de emergência podem auxiliar na melhora das estratégias para tratar esses pacientes e ampliar o conhecimento da fisiopatologia do TCE nas primeiras horas após o evento traumático. O objetivo desta investigação foi analisar a evolução das vítimas de TCE contuso durante a permanência na sala de emergência. Materiales y métodos: Estudo do tipo coorte prospectivo, com avaliação das vítimas na admissão na sala de emergência e 2, 4 e 6 horas após. Participaram desta pesquisa 46 vítimas que apresentaram TCE contuso como lesão principal, com idade -15 anos. A evolução das vítimas foi descrita pelas diferenças nas pontuações do rapid emergency medicine score (REMS). Resultados: Entre a admissão e 2 horas após, foram observadas mudanças desfavoráveis em 35,1\% das vítimas, entre 2 e 4 horas, em $13,6 \%$ e entre 4 e 6 horas, em $42,8 \%$ dos casos; a melhora foi observada entre $27 \%$ e $28,6 \%$ da casuística. Após 2 horas da admissão na sala de emergência, o escore da escala de coma de glasgow (ECGI) e a saturação periférica de oxigênio (SpO2) ficaram estáveis e as alterações desfavoráveis após esse período ocorreram quase que exclusivamente na frequência cardíaca (FC) e pressão arterial média (PAM). A melhora de mais de $25 \%$ das vítimas ocorreu em todas as avaliações, porém a frequência de evolução desfavorável foi maior do que favorável entre admissão e 2 horas e essa diferença foi mais acentuada após 4 horas. Conclusiones: Houve evidências que a permanência superior a
4 horas na sala de emergência foi desfavorável para as vítimas e que os parâmetros FC e PAM foram os de mais difícil controle durante a permanência das vítimas na sala de emergência.

Mecanismo De Trauma No Idoso. Mário L Quintas, Vitória S Oliveira, Camila C Silva, Nathalia S Oliveira, Camila M Kawata, Beatriz T Carvalho, Gustavo L Neves, Matheus ERF Souza, Lorayne AC Pereira, Gustavo G Quintas. From the Hospital Estadual Vila Alpina - Seconci-Oss, São Paulo, Brazil.

Introducción: Apresentar as características peculiares do idoso traumatizado, seus mecanismos e padrão das lesões. O objetivo é analisar vítimas de trauma de causas externas com idade acima de 60 anos, levando-se em conta o tipo de acidente e sua evolução e o tempo de reabilitação, atendidos no Hospital Estadual Vila Alpina - SECONCI-OSS, hospital de nível secundário localizado na zona Leste de São Paulo. Materiales y métodos: Estudo retrospectivo dos atendimentos nos meses deoutubro, novembroedezembrode2018,devítimas detrauma com 60 anos ou mais, avaliando-se prevalência quanto ao sexo, faixa etária, mecanismo de trauma, tipo de lesão, atendimento e evolução. Resultados: Foram atendidos pela equipe cirúrgica no período 4.182 pacientes, sendo $34,8 \%$ vítimas de trauma, compreendendo $8,9 \%$ na faixa de 60 a 75 anos com prevalência do sexo feminino, $71,2 \%$ em relação ao masculino, $28,8 \%$, predominando a queda da própria altura, $41,7 \%$, queda da escada, 23,2\%, FAB, 17,3\%, queda de laje, $7,1 \%$, colisão e atropelamento, $10,7 \%$. Na faixa dos 75 aos 102 anos, $5,6 \%$ vítimas, prevalecendo o feminino, $80 \%$ em relação ao masculino, $20 \%$, com queda da própria altura, $64 \%$, queda de altura, $12 \%$, queda da escada, $12 \%$, colisão e atropelamento, $10 \%$, queimadura $2 \%$. Conclusiones: A maior incidência de trauma no idoso observado no período foi queda da própria altura, prevalecendo o sexo feminino, preferencialmente na própria residência ou no entorno, relacionado as atividades diárias rotineiras. As características fisiológicas próprias do idoso, como a diminuição da capacidade visual e auditiva, efeitos colaterais do uso crônico de medicações, doenças mentais degenerativas, diminuição da capacidade de concentração facilitam o trauma na faixa etária em questão, devendo-se ter em mente todos os fatores de prevenção, pois nesse tipo de trauma temos uma recuperação mais lenta e necessidade maior de reabilitação.

Implementación De Taller De Primeros Auxilios Y Evaluación De Necesidad De Reeducación, En Adolescentes Entre 13 Y 15 Años. María JJ Torres, Ana IC Guillén, Juan CS Molina. From the Universidad del Azuay, Cuenca, Ecuador.

Introducción: Diariamente nos enfrentamos a situaciones de emergencia en una gran variedad de escenarios. Las primeras causas de mortalidad a nivel mundial incluyen las enfermedades cardiovasculares, que podrían manifestarse como parada cardíaca. Además, el atragantamiento se encuentra entre las primeras causas de muerte accidental. La población debería estar capacitada para activar el sistema de emergencia y brindar los primeros auxilios en caso de ser requerido, por lo que su enseñanza debería iniciarse a edades tempranas y mantenerse de manera constante, esto aumenta de manera notable la supervivencia de la víctima. Materiales y métodos: Se realizó un estudio longitudinal, prospectivo de intervención educacional, con reevaluación teórica 
en un periodo de 12 meses; a estudiantes entre 13-15 años de colegios particulares de la ciudad de Cuenca, Ecuador. Se impartió un taller teórico - práctico con duración de 80 minutos, con evaluación previa, posterior inmediata y posterior a largo plazo. Resultados: Se trabajó con un grupo sin conocimientos previos en primeros auxilios, con características físicas similares a las de un adulto. Existe diferencia estadísticamente significativa ( $p$ ). Conclusiones: La enseñanza de primeros auxilios debería ser implementado dentro de la malla curricular de la educación de adolescentes y niños capacitados tendremos adultos listos para responder frente a situaciones de emergencia. La capacitación debería ser constante, en un periodo menor a un año, por la decadencia de conocimientos básicos y destrezas.

Two Cluster Model for the Transition from Acute to Chronic Pain in Traumatically Injured Patients. Colleen Trevino, Timothy Geier, Cecilia Hillard, Karen Brazel, Terri. From the Medical College of Wisconsin, Milwaukee, USA.

Introduction: Pain on hospital discharge is a significant predictor for the development of chronic pain in traumatically injured hospitalized patients. However, the longitudinal structure of this transition has not been defined. The trajectory of chronic pain development and the predictors of those trajectories needs to be determine in order to truly understand this transition. Materials and methods: A secondary analysis of a prospective longitudinal cohort of participants admitted to a Midwestern American level 1 trauma center post traumatic injury was completed using the mean pain ratings at hospital discharge and 6-month follow up time points. Ward's method for hierarchical cluster analysis was completed. Results: A two-group cluster was identified in which patients with severe pain at hospital discharge continued to have severe chronic pain and those who had less severe pain at hospital discharge did not have chronic pain. The mean pain score on discharge for those developing chronic pain was $7.2(\mathrm{SD}=2.1)$ and $6.4(\mathrm{SD}=1.9)$ at 6 months. The mean pain score on discharge for those whose pain resolved was $4.7(S D=2.5)$ and $1.1(S D=1.4)$ at 6 months. Conclusion: Traumatically injured patients who continue to have severe pain on discharge are at high risk for the development of severe chronic pain related to their injuries. Trauma providers must focus on improving pain control before hospital discharge to mitigate the possible development of chronic pain after injury.

Assessing Patient Surgical Safety as a Key Component of Quality of Care in Manaus, Amazonas. Jessica Correia, Thais Oliveira, Marcela Catunda, Jania Ramos, Isabelle, Paul Truche, Lina Roa, José E Souza, Rodrigo Vaz. From the Universidade do Estado do Amazonas, Amazonas/Manaus, Brazil.

Introduction: Low and middle income countries (LMICs) perform only $6.3 \%$ of the world's surgical procedures, but experience two-thirds of adverse healthcare events. Despite a universal health care system in Brazil, the state of Amazonas struggles to evaluate surgical care with respect to safety and quality. Our objective is to assess patient safety among the domains of structure, process and outcomes among patients undergoing surgeries in Manaus, Amazonas. Materials and methods: This is a prospective observational study of patients undergoing abdominal surgery at Hospital e Pronto Socorro 28 de Agosto in Manaus between July and December 2017.
Patient safety was evaluated using the Donabedian framework which measure quality in three domains: structure, process and outcome. Safe structure was evaluated via administrative data on morbidity and mortality meetings. Safe process was evaluated through direct observation of the use of the surgical checklist in patients undergoing abdominal surgery. Safe outcome was measured through in-hospital peri-operative mortality rate (POMR). Comparisons between emergent and elective surgical safety were performed using chi squared test. Results: Regarding safe structure, the hospital does not hold mortality and morbidity meetings. For safe process, a total of 181 surgeries were observed (20.2\% electives and $79.78 \%$ emergent). The surgical checklist was not performed completely in any observed surgical cases. Pulse oximetry was used in $100 \%$ of cases, however, a name band check was never performed. Peri-operative antibiotic adminstration was performed in 69\% (124) of cases, with improved use in emergency cases (79\%) vs elective (43\%) (P). Conclusion: There are barriers to patient safety among all three domains. The use of this novel patient safety tool can be used to assess baseline surgical safety in low resource settings. Opportunities for quality improvement include starting morbidity and mortality meetings and improved surgical checklist use.

Traumatismo Toracoabdominal Penetrante: Que Cavidad Operar Primero? Guillermo Barillaro, Celeste Echavarria, Sofia Bou, Cristian Assel, Favio, Juliana Nazaretto, Andrea Potes. From the Hospital Interzonal General De Agudos Dr Oscar Alende, de Mar Del Plata, Mar Del Plata, Provincia De Buenos Aires, Argentina.

Introducción: Los pacientes con traumatismos toracoabdominales penetrantes (TTAP) por arma de fuego (HAF) o cortopunzante (HCP) plantean el desafío de decidir que cavidad operar prioritariamente. El objetivo del trabajo fue analizar características de estos pacientes con TTAP con secuencia de intervenciones quirúrgicas combinadas, error por inapropiada secuencia de dichos procedimientos y relación de esos factores anteriores con mortalidad. Materiales y métodos: Revisión de historias clínicas de pacientes asistidos en nuestra institución entre enero del 2005 y diciembre del 2018, con TTAP los cuales requirieron procedimientos operatorios tanto en el tórax como en el abdomen. Resultados: Fueron asistidos 79 pacientes con TTAP, 48 con normalidad hemodinámica (grupo I) y 31 con shock hipovolémico (grupo II). En el grupo I predominaron las HCP (40) sobre HAF (8), y las lesiones del lado izquierdo (42) $(87,5 \%)$. En este grupo no hubo errores en el manejo quirúrgico secuencial ni se registró mortalidad. En el grupo II predominaron las HAF transfixiantes de la línea media. En 8 casos se registró error al abordar primero la cavidad con lesiones menos graves, falleciendo 7 de ellos y determinando mortalidad de $25,8 \%$ para el grupo II. El análisis del error en esos 8 casos hallo que estuvo relacionado con resultados erróneos de la ecografía y/o subestimación del débito del drenaje pleural y de la radiografía de tórax post-drenaje pleural. Conclusiones: Los pacientes con TTAP y hemodinámicamente compensados presentaron predominio de HAB del lado izquierdo y no tuvieron errores en el manejo ni mortalidad. En aquellos con TTAP y shock, predominaron las HAF transfixiantes de la línea media, y este grupo presento los errores de manejo y mortalidad. La mayoría de esos errores fueron considerados potencialmente prevenibles y se relacionaron con resultados falsos de la ecografía y 
con subestimación del débito del drenaje pleural y de la radiografía de tórax post-drenaje pleural.

Actualización Y Análisis De Una Serie De Trauma Renal En Pacientes Pediátricos Menores De 15 Años En Un Centro Hospitalario De Alta Complejidad De La Ciudad De MedellínColombia. Carlos AD López, Julieta C Restrepo, Carlos HM Uribe, David AM Toro. From the Universidad de Antioquia, Antioquia/ Medellín, Colombia.

Introducción: Las lesiones renales representan del 5-10\% de los órganos afectados en el traumatismo de abdomen. El método diagnóstico ideal es la tomografía y cada vez se propende por la búsqueda de estrategias de manejo minimamente invasivas teniendo en cuenta que la mayoría de pacientes se benefician de la observación clínica. Materiales y métodos: Estudio retrospectivo y descriptivo. Se incluyeron pacientes menores de 15 años ingresados al servicio de urgencias pediátricas con sospecha $\mathrm{y} / \mathrm{o}$ diagnóstico de trauma renal entre enero 2013 y marzo de 2019 Resultados: Se identificaron 144 pacientes con diagnóstico de trauma abdominal y sospecha de compromiso renal; en el $29.9 \%$ se pudo confirmar el diagnóstico. De los 43 pacientes, el $65.1 \%$ fueron hombres y la edad promedio fue de 9.4 años. El trauma cerrado correspondió al 93\% siendo la principal etiología las caídas (27.9\%) seguidas del trauma contuso (20.9\%). Los síntomas principales fueron el dolor lumbar, abdominal y la hematuria. El trauma grado III fue el más frecuente (40.2\%) seguido del IV (30.2\%). La conducta más frecuente fue la observación clínica $(76.7 \%)$ con una efectividad del 100\%. El 16.3\% requirió cirugía y el $7 \%$ angioembolización como manejos iniciales con una efectividad del $100 \%$ y $66 \%$ respectivamente. La tasa de complicaciones fue del $7 \%$ y no se presentaron muertes. Conclusiones: Durante el tiempo de recolección se identificaron cambios importantes en la epidemiología del trauma pero conservando la relación según los grados del mismo. Llama la atención el papel de las heridas por arma cortopunzante en esta segunda etapa de recolección. La observación clínica continúa siendo la estrategia más utilizada inclusive en grados IV y V con buenos resultados y bajas tasas de complicaciones. El tiempo de estancia hospitalaria no parece estar relacionada con el grado del trauma. Se requieren más estudios para realizar conclusiones de mayor poder epidemiológico.

Comparison of Patient Satisfaction among Elective and Emergent Surgical Patients in Amazonas, Brazil Using the Hospital Consumer Assessment of Healthcare Providers and Systems Survey. Yasmin BS Gomes, Nicolas B Cavalcanti, Pedro H Da Silva Gomes, Jania A Ramos, Lina, Isabelle Citron, Paul Truché, Rodrigo $V$ Ferreira, José ED Santos Souza. From the University of State of Amazonas, Manaus, Brazil.

Introduction: The service provided to a patient is important to evaluate quality of care offered. In the state of Amazonas, satisfaction among surgical patients is understudied and represents an opportunity to identify areas for quality improvement. The aim of this study is to evaluate satisfaction of patients undergoing abdominal surgery in Manaus, Amazonas. Materials and methods: A prospective, observational study of patients undergoing abdominal surgery was performed from July to August 2017 at a tertiary care hospital in Manaus. Patients were interviewed using an adapted version of the Hospital Consumer Assessment of HealthCare Providers (HCAHPS) tool. Satisfaction was scored along 5 composites (doctor communication, nurse communication, staff responsiveness, pain management, medication communication), 2 discharge questions (discharge instructions, follow-up instructions), 2 individual measures (hospital cleanliness, hospital quietness) and 2 global items (overall satisfaction, willingness to recommend the hospital). Scores were compared between patients undergoing emergent and elective surgery. Results: 126 patients were interviewed of which 99 (79\%) underwent emergent surgery and 26 (21\%) underwent elective surgery. Average patient satisfaction was 2.9/4 for composites, 2.8/4 for individual items and 6.1/10 for global items. The highest scores were noted with overall hospital rating (8.7/10), doctor communication (3.8/4) and nurse communication (3.8/4). The lowest scores were noted for staff responsiveness (2.3/4) and hospital cleanliness (2.29/4). Among elective patients, satisfaction was significantly higher for doctor communication (3.76 vs $3.96 p=0.02$ ), hospital quietness (3.18 vs $3.65 p=0.03$ ) and overall hospital rating (8.58 vs $9.31 p=0.001)$. Only $45(36 \%)$ patients reported receiving complete discharge instructions with elective surgical patients more likely to receive discharge instructions $(26 \%$ vs $73 \% p<0.0001)$. Conclusion: Overall patient satisfaction with surgical care is high, but elective patients have significantly higher overall satisfaction compared to emergent patients and higher rates of completed discharge instructions. Patient satisfaction surveys can be implemented in low-resource settings and can guide improvement of surgical quality.

Análisis Del Trauma Por Caídas: Estudio Del Registro De Trauma Del Hospital Interzonal General De Agudos San Roque De Gonnet, Buenos Aires Argentina. Fabiana Ferrero, Graciela Badin, Sandra Aronne, Analía Emer, Agustin, Manuela Alvarez, Juan $P$ Grillo. From the Hospital Interzonal General de Agudos San Roque de Gonnet, La Plata, Argentina.

Introducción: $60 \%$ de las consultas del Servicio de Emergencias corresponden a trauma. Del resto de los casos, las caídas son uno de los mecanismos más frecuentes, incluyendo las caídas de altura y en un mismo nivel de origen laboral y domiciliario. Objetivos: describir características del trauma por caídas como mecanismo lesional y analizar escores de severidad determinando la calidad de atención en nuestro hospital. Materiales y métodos: Estudio descriptivo retrospectivo. Fuente: Registro de Trauma de la Fundación Trauma. 2521 hechos de 30/06/2010-28/02/2019. Variables: Clínicas-epidemiológicas (edad, sexo, mecanismo lesional, tipo de trauma, tipo de lesión)y de Calidad de atención (RTS, ISS, TRISS, tipo de egreso). Se incluyeron traumatizados internados $>24$ horas, fallecidos o derivados. Resultados: 354 casos correspondieron a caídas (14\%), $3^{\circ}$ lugar luego de colisiones por moto $(27,7 \%)$ y agresiones $(15,7 \%)$, aumento $>3$ veces en últimos 2 años. Mayor frecuencia en hombres (62,7\%) entre $75-84$ años (18\%). 45\% son caídas en mismo nivel y $14 \%$ de altura. 52\% con comorbilidades, presentando el 33\% >2. 96\% trauma contuso. El sitio lesional más frecuente es la cabeza (25\%). Según ISS, 84\% leves y moderados, mayor mortalidad en severos y críticos. $45 \%$ de fallecidos son críticos y $10 \%$ severos. Sobrevida por TRISS acorde al estándar: O/E: 1,01. 81\% sin complicaciones. 5,6\% 
ingresaron a UTI, estadía de 16,55 días. Estadía hospitalaria de 7,2 días. Egreso: alta médica (68\%) y derivaciones (18\%). Mortalidad $8 \%$, predominando hombres $(66 \%) ;>55$ años $(72 \%)$; lesiones más frecuentes: cerebrales focales $(51,7 \%)$ y fracturas de fémur (41\%). Conclusiones: Las caídas son causa frecuente de mecanismo lesional, ocupando el $3^{\circ}$ lugar. Mortalidad mayor en grupos de severidad más altos. Probabilidad de sobrevida alta. La mayoría de los ingresos se deben a: lesión cerebral focal, fractura de fémur y concusión cerebral, siendo las principales causas de muerte las dos primeras. No hay registro de uso de elementos de seguridad. El uso del registro permite orientar campañas de prevención, dirigidas al uso de elementos de protección en trabajadores y a la educación del adulto mayor.

Hemoglobina En Trauma Craneoencefálico Severo a Gran Altitud. Antonio Viruez, Jorge Jiris, Jorge Antezana, Félix Cáceres. From the Centro De Trauma Hospital Corazon De Jesus, El Alto, Bolivia.

Introducción: Los residentes de gran altitud tienen un nivel de hemoglobina más alto a comparación de residentes a nivel de la costa, lo cual, según alguna literatura se correlaciona a un mayor daño en diferentes órganos, especialmente a nivel del sistema nervioso central. El objetivo del presente estudio fue evaluar el efecto de la concentración de hemoglobina de ingreso en la mortalidad en pacientes residentes a 4090 metros sobre el nivel del mar con trauma craneoencefálico severo. Materiales y métodos: Estudio retrospectivo transversal descriptivo. Se incluyeron en el estudio pacientes residentes de gran altitud al menos durante los últimos 6 meses, sin antecedentes de enfermedad cardiopulmonar, sin hábito tabáquico, con el diagnóstico de trauma craneoencefálico severo (escala de coma de Glasgow-8) quienes fueron clasificados según el nivel de hemoglobina al ingreso en baja (menor a $11.9 \mathrm{~g} / \mathrm{dL}$ ), moderada (12-14.9 g/dL) yalta (mayor a $15 \mathrm{~g} / \mathrm{dL}$ ). Se creó una base de datos en el programa estadístico SPSS versión 18 y realizó el análisis estadístico mediante medidas de resumen, tendencia central y dispersión así como prueba estadística mediante t de Student. El estudio cuenta con la aprobación del Comité de Bioética en Investigación. Resultados: La muestra estuvo conformada por 72 pacientes, $78 \%$ del sexo masculino, media de edad 47 años, tiempo de internación media 132 horas, $85 \%$ de supervivencia, el nivel medio de hemoglobina entre supervivientes y fallecidos es del 13.48 $\mathrm{g} / \mathrm{dL}$ y $13.46 \mathrm{~g} / \mathrm{dL}$, respectivamente ( $p 0.49$ ), el $59 \%$ de pacientes supervivientes presentaron niveles de hemoglobina entre $12-15 \mathrm{~g} / \mathrm{dL}$. Conclusiones: Se debe considerar el mantener niveles de hemoglobina entre $12-15 \mathrm{~g} / \mathrm{dL}$ como objetivo terapéutico en pacientes residentes de gran altitud que sufren de trauma craneoencefálico severo. Valores más elevados podrían asociarse a resultados desfavorables.

Standardization of Prehospital Care in Kigali, Rwanda. Ashley Rosenberg, Megan Wojick, Jackie Mukagasasira, Mediatrice Niyonsaba, Myles, Jean M Uwitonze, Igance Kabagema, Theophile Dushime, Sudha Jayaraman. From the Virginia Commonwealth University Health Systems, Richmond, Virginia, USA.

Introduction: Non-communicable diseases (NCDs) and injuries present a substantial burden in low- and middle-income countries
(LMICs). However, many of these deaths may be prevented by high quality prehospital and emergency care. We aim to develop standardized checklists and protocols for common conditions managed as well as develop a data management system by service d'aide medicale d'Urgence (SAMU), the prehospital emergency medical service in Kigali, Rwanda. Materials and methods: We identified the six most common emergencies treated by SAMU in Kigali, Rwanda using a preexisting electronic database. In collaboration with SAMU, we developed protocols and checklists based on established international best practices. Stakeholder analysis allowed for the protocols to be context appropriate. Following this, we created a data collection sheet and electronic database and dashboard to monitor and evaluate clinical performance. Results: The six identified emergencies included extremity injury, traumatic brain injury, altered mental status/stroke, diabetes complications, and both pediatric and adult respiratory issues. We created checklists for each protocol consisting of four to six quality improvement metrics. The leadership of SAMU at the Ministry of Health of Rwanda approved the checklists as the national standard for prehospital care. A data collection sheet with embedded QI metrics and a data management system with built in dashboard were implemented. Evaluation of clinical performance based on these checklists is currently in progress. Conclusion: There is scant literature on establishing high-quality prehospital services in LMICs. With minimal investment, we were able to identify common prehospital emergencies and develop and implement standards of care to ensure high-quality prehospital services. Standardizing treatments for common emergencies are likely to improve patient outcomes and address the current burden of NCDs and injuries in LMICs. Further work is needed to evaluate the implementation of the checklists in Rwanda.

Standardization of Surgical Morbidity and Mortality Conferences: Development and Dissemination of a "Toolkit". Lacey Lagrone, Leah Isquith-Dicker, Manuel R Castro, Roberto Valderrama, Juan Jaime, Erica Dextre, Giani Aragon, Charles Mock, Eduardo $H$ Egoavil. From the University of Washington, Washington, United States.

Introduction: Objective: This project aimed to establish nationallevel consensus on a standard for surgical morbidity and mortality (M\&M) conferences, and facilitate diffusion of this standard via a practical, simple, M\&M "toolkit." Methods: A multidisciplinary group of surgical and trauma care providers were invited to participate in a "working group" under the auspices of the quality chapter of the peruvian general surgery society. Materials and methods: Through these meetings four toolkit components underwent a total of five revisions over eight weeks. Toolkit component \#1: Guide for planning M\&M conferences. This document included sections on conference logistics, and case selection, presentation, discussion, documentation and follow-up. The guide includes evidence-based recommendations regarding M\&M conference best-practices. Toolkit component \#2: Form for documenting M\&M conferences. This document provides a record of the discussion, prompts conference participants to complete all essential components of M\&M discussion, and provides a place to document planned corrective actions. Results: Toolkit component \#3: Template for case presentations. 
This template was designed to assist the junior clinician, often a resident, in selecting what information is salient in developing a concise case presentation which includes all information necessary to inform a discussion of preventability and root cause. Toolkit component \#4: Code of conduct Phase 1 research suggested that interpersonal conflict was the etiology of many failed attempts or non-sustainable M\&M conferences in Lima, Peru. One suggested response to this common problem was the development of a "code of conduct" which would be signed by all participants and posted in a visible, central location. Conclusion: We successfully established a toolkit for national dissemination of a standardized surgical/emergency $M \& M$ conference. Patient outcome data are needed to confirm efficacy, and once established, reproduction on larger scale and in other country and specialty contexts may be warranted.

$\mathrm{PaCO}_{2}$ En Trauma Craneoencefálico Severo a Gran Altitud. Antonio Viruez, Jorge Jiris, Jorge Antezana, Felix Caceres. From the Centro De Trauma Hospital Corazon De Jesus, El Alto, Bolivia.

Introducción: Existe una importante confusión en la terminología, ya que a lo que la literatura usualmente se refiere como hiperventilación es de hecho, hipocapnia, el cual conlleva importantes efectos a nivel del flujo sanguíneo cerebral. El objetivo del presente trabajo es describir los valores de $\mathrm{PaCO}_{2}$ en pacientes con trauma craneoencefálico severo a 4050 metros sobre el nivel de mar y su desenlace clínico. Materiales y métodos: Estudio retrospectivo transversal descriptivo. Se incluyeron todos los pacientes ingresados a la Unidad de Cuidados Intensivos del Centro del Trauma "Hospital Corazón de Jesús" de la ciudad de El Alto a 4090 metros sobre el nivel del mar dentro del periodo 01 de marzo de 2017 a 31 de marzo de 2019, residentes de gran altitud al menos durante los últimos 6 meses, con el diagnóstico de trauma craneoencefálico severo (escala de coma de Glasgow-8). Se creó una base de datos en el programa estadístico SPSS versión 18 y realizó el análisis estadístico mediante medidas de resumen, tendencia central y dispersión así como la prueba estadística t Student. El estudio cuenta con la aprobación del Comité de Bioética en Investigación. Resultados: La muestra estuvo conformada por 72 pacientes, $78 \%$ del sexo masculino, media de edad 47 años, $85 \%$ de supervivencia, el nivel medio de $\mathrm{PaCO}_{2}$ entre supervivientes y fallecidos es del 22.49 $\mathrm{mm} \mathrm{Hg}$ y $30.82 \mathrm{~mm} \mathrm{Hg}$, respectivamente ( $p$ menor a 0.05 ), el $82 \%$ de pacientes supervivientes se encontraron en hiperventilación moderada según la clasificación propuesta para gran altitud. Conclusiones: Los valores de hipocapnia e normocapnia asociados a evolución favorable en el presente estudio, son menores a los valores de referencia consultados en la literatura a niveles menores de altitud.

Evaluation of Simulation-based Training Curriculum for Physicians in China. Eugenia Lee, Saskya Byerly, Valerie Hart, Ruiz Gabriel, Antonio. From the University of Miami/Ryder Trauma Center/Jackson Memorial Hospital, Miami, Florida, USA.

Introduction: Simulation-based training is an effective educational resource for physicians without access to large traumatically injured patient volumes or formal training in trauma management. As China continues to industrialize, the mortality from injury is increasing, making traumatic injury the fifth most common cause of death. China's trauma system is still developing and there is no organized training for providers. In order to address injury-related mortality in a nascent trauma system, efficient and effective means of education must be utilized. Methods: A 20-question exam was administered to trauma care providers in Shanghai and Beijing on the management of trauma patients. A 2-day simulation-based course with 16 trauma scenarios was administered. Participants met at a single center in Shanghai for the training, while the course was administered to in-house staff in Beijing. The curriculum included the ABC's of trauma, simulation-based case studies, debriefing, radiological discussion, as well as discussion of guidelines and protocols. A post-instruction exam was then administered. Results of pre- and post- tests were then compared using students $t$ - test. Results: A total of 35 physicians and medical staff (23 Shanghai and 12 Beijing) participated in this study. Of the 23 participants in Shanghai, 20 were medical doctors and 3 were ancillary medical professionals. All 12 participants in Beijing were medical doctors. Pre-test and post-test means in Shanghai were $57 \%$ and $79 \%$ respectively $(p<0.001)$. Pre- and post-test means in Beijing were $38 \%$ and $71 \%$ respectively $(p<0.001)$. Combined pre- and post-test means were 51 and $77 \%$ respectively $(p<0.001)$. Conclusion: Simulation-based training is an effective and efficient method to educate trauma providers. This curriculum can be useful in various educational settings for current providers as well as medical students and residents in training.

Vena Cava Inferior Como Factor Predictor Del Shock En Trauma: Medición Ecográfica. Adalid Gonzalez, Fernando Rey, Pablo Ottolino, Erika Vasquez, José, Luis Richard, Yovany Contreras, Roberto Guillen, Cristopher Varela, Adrian Terán. From the Hospital General del Este “Dr Domingo Luciani”, Caracas, Dc, Venezuela.

Introducción: El diagnóstico preciso del shock es un reto para el cirujano. En nuestro centro frecuentemente existen varios pacientes hemodinámicamente estables esperando quirófano para control del sangrado, debiendo priorizarlos muy eficazmente. Objetivo: Establecer la medición ecográfica del diámetro de la vena cava inferior como factor predictor del shock en pacientes politraumatizados. Materiales y métodos: Estudio de corte transversal para determinar la medición ecográfica de la vena cava inferior a 40 pacientes que ingresaron a la unidad de politraumatizados (UPT) del Hospital General del Este "Domingo Luciani", Caracas, entre enero y abril de 2018, seleccionando 2 grupos; el grupo I: pacientes en shock, con tensión arterial sistólica menor a $90 \mathrm{~mm} \mathrm{Hg}$ al ingreso, y el grupo: pacientes controles con cifras tensionales normales. El estudio de imagen de la vena cava inferior se realizó con el equipo de ecografía ALOKA prosound SSD-?5 y con el ultrasonido portátil MicroMaxx SonoSite, en el cual se midió índice de colapsabilidad. Se registró en instrumento de recolección de datos, previamente validado. Análisis estadístico con la prueba de $t$ de student para muestras independientes y valoración de puntos de corte diagnóstico realizada con la prueba de ROC. Resultados: Rango de edad de 19 a 58 años en el grupo control y de 21 a 45 años en el grupo de shock. La media del Índice de colapsabilidad (IC) de la VCI para el grupo control y de shock fue de $26 \pm 12,7 \%$ y de 58,5 $\pm 5,9 \%$, respectivamente; El índice de colapsabilidad fue $>50 \%$ en todos los pacientes del grupo de shock. 
Conclusiones: Conclusiones: La medición del diámetro de la VCI es un predictor de shock, siendo el IC el parámetro más sensible y especifico. Recomendamos el entrenamiento del residente de Cirugía general en éstas mediciones para optimizar la atención del paciente severamente traumatizado.

Impacto Del Programa De Formación Nacional Específico En Cirugía De Urgencias Para Residentes De La Asociación Española De Cirujanos. Virginia MD Muñoz-Cruzado, Luis T Aguilar, José AL Ruiz, Andrea C Serra, Ignacio, Dieter M García, Aitor L Olavarria, Jose MA Narvaez, Felipe P Ciuró. From the Asociación Española de Cirujanos, Madrid, Spain.

Introducción: La necesidad de una formación específica en cirugía de urgencias a nivel nacional se ha hecho patente desde hace años. Más del 35\% de las cirugías realizadas en nuestra especialidad son cirugías urgentes. Sin embargo, hasta 2018 no se crea el primer Curso de Cirugía de Urgencias para Residentes de la Asociación Española de Cirujanos. Nuestro objetivos es evaluar el impacto de este curso sobre la formación de los residentes. Materiales y métodos: Análisis descriptivo de los resultados de los test de evaluación pre y post curso, así como de la encuesta de satisfacción de los alumnos realizados durante el año 2018. Resultados: Durante el año 2018 se han realizado 10 cursos en distintas ciudades españolas. El número de residentes formados fue 112. El número de residentes que completaron el examen pre y postcurso fue 91. La puntuación media en el examen precurso fue $6.29 \pm 1.57$ y $8.08 \pm 1.39$ en el postcurso (sobre una valoración máxima de 10 puntos). Las expectativas de los estudiantes fueron 8.83/10. Al 57 '28\% de los participantes le pareció muy bien el contenido del curso y al $42.72 \%$ bien. La metodología fue valorada como buena o muy buena por el $\mathbf{9 7 . 0 9 \%}$ de los participantes. La aplicabilidad del curso se puntuó como mucha o bastante en $98.06 \%$ y regular en $0.97 \%$. La duración del curso se considera normal por el $89.32 \%$, escasa por el $8.74 \%$ y excesiva por el $0.97 \%$. La documentación entregada a los participantes fue valorada como buena o muy buena en el $75.93 \%$, regular en el $15.53 \%$ y mala o muy mala en el $7.76 \%$. Conclusiones: La formación específica en cirugía de urgencias consigue mejorar el conocimiento de los residentes de cirugía en esta área específica y es valorada de forma muy positiva por los mismos.

¿Están Los Cirujanos Españoles Preparados Para Atender a Los Pacientes Politraumatizados? Virginia MD Muñoz-Cruzado, Felipe P Ciuró, Maria DP Díaz, Soledad M Condón, Isidro, Fernando T Fuentes, Jose MA Narváez, LT Aguilar From the Asociación Española de Cirujanos, Madrid, Spain.

Introducción: El entrenamiento específico en el manejo del paciente politraumatizado es esencial para el cirujano. La formación mediante cursos específicos en esta materia impacta de forma directa en la atención de estos pacientes. El objetivo de este estudio es conocer cómo es la formación específica en la atención del paciente politraumatizado de los cirujanos españoles. Materiales y métodos: Se ha realizado una encuesta nacional administrada a los cirujanos miembros de la Asociación Española de Cirujanos. En ella se ha evaluado su grado de participación en la Cirugía de Urgencias, y por tanto la posibilidad de atender pacientes politraumatizados, su valoración sobre la atención del paciente politraumatizado en su centro, así como su formación específica.
Resultados: La encuesta ha sido completada por 511 cirujanos (10.26\%) de 27 provincias españolas siendo las Comunidades Autónomas más participativas Andalucía y Cataluña. 453 de los encuestados realizan guardias (88.65\%). Tan solo 171 (33.46\%) refieren tener un registro de pacientes politraumatizados en su hospital. El $87.48 \%$ de refieren que en su hospital el cirujano general no está implicado en la atención del trauma grave desde el momento inicial. El $66.47 \%$ ha realizado el curso ATLS, el $40.78 \%$ el DSTC y el $11.57 \%$ el MUSEC (7.25\% otro curso sobre E-FAST). $14.51 \%$ se encuentran en lista de espera para realizar el curso ATLS y $7.25 \%$ para el DSTC. Solamente el $43.33 \%$ considera la atención al trauma grave en su hospital como mala o deficiente. Conclusiones: La formación específica en el tratamiento del paciente politraumatizado es aún insuficiente en España y con muchos aspectos susceptibles de mejorar. En la mayoría de los hospitales, el cirujano no se implica en la atención inicial del politramatizado grave. A pesar de esto, un gran porcentaje de cirujanos trabaja en urgencias habitualmente y potencialmente se enfrenta al reto del manejo de estos pacientes.

Nuevo Abordaje Quirúrgico Para El Tratamiento De La Fístula Enteroatmosférica: El Colgajo Anterolateral De Muslo. Virginia MD Muñoz-Cruzado, Luis T Aguilar, Araceli L Borrego, Fernando B Pulido, Alejandro, María JT López, Daniel A Sánchez, Eduardo P Del Pozo, Javier $P$ Ruiz, Felipe $P$ Ciuró. From the Hospital Universitario Virgen del Rocío, Sevilla, Spain.

Introducción: Cuando aparece una fístula enteroatmosférica (FEA), el cirujano se encuentra principalmente con 2 problemas: la propia fístula en sí y la pérdida importante de tejido que asocia habitualmente en su periferia. Una buena solución parece ser la cobertura del defecto de pared con tejido sano. El objetivo de este trabajo es describir la técnica del colgajo anterolateral de muslo en el tratamiento de la FEA. Materiales y métodos: Estudio descriptivo retrospectivo de 4 casos a los que se aplica esta técnica en el periodo Mayo'15-Noviembre'18. Descripción de las características basales, del tipo de FEA y de los resultados obtenidos. Resultados: La edad de los paciente fue entre 16 y 83 años (media 50.5 DS 27.88); dos eran mujeres. Las causas iniciales que llevaron a los pacientes a múltiples cirugías y el desarrollo de la complicación fueron un vólvulo, una dehiscencia, un carcinoma de recto perforado y una diverticulitis perforada con peritonitis difusa. Las medidas iniciales del defecto fue de 8 a $14 \mathrm{~cm}$ transversal (media 12.5 DS 3.42 ) y 16 a $22 \mathrm{~cm}$ longitudinal (media 18.25 DS 2.63). Una fístula era a nivel de yeyuno y las otras tres a nivel de ileon. El débito era alto (> 500 cc/día) en un paciente, medio en otro (250-500cc/día) y bajo. Conclusiones: El uso de los colgajos anterolaterales de muslo en el tratamiento de la FEA puede ser un método alternativo que garantiza la cobertura y reconstrucción del defecto proporcionando un tejido adecuado para el cierre de la misma.

Análisis Del Impacto Del Plan De Prevención Nacional Contra La Violencia De Genero “Por Una Vida Libre De Violencia De Género, Con Mirada Generacional". Virginia MD Muñoz-Cruzado, Luis T Aguilar, Alejandro S Arteaga, María JT López, Jose, Daniel A Sánchez, Eduardo P Del Pozo, Antonio N Infante, Javier P Ruiz, Felipe $P$ Ciuró. From the Hospital Universitario Virgen del Rocío, Sevilla, Spain. 
Introducción: Debido a la alta cifra de mujeres lesionadas por violencia de género, es fundamental consolidar un plan de acción que permita prevenir, reducir y reparar este tipo de violencia. El objetivo de nuestro estudio es analizar el impacto del plan de prevención contra la violencia de género 2016-2019 que se lleva a cabo actualmente España. Materiales y métodos: Análisis descriptivo de los datos recogidos en los registros nacionales sobre violencia de género desde Enero de 2013 a Diciembre de 2018. Comparación de resultados previos y posteriores a la implantación del plan. Resultados: Tras la publicación y aplicación del plan, el número anual de víctimas mortales por violencia de género ha disminuido levemente pasando de una media de 53 muertes anuales entre los años 2013-2015 a 49 entre los años 2016-2018. La nacionalidad española es la más frecuente entre los agresores. La media anual de huérfanos por violencia de género en 2013-2015 fue de 45.3 mientras que en 2016-2018 descendió a 31.3. El porcentaje de sentencias condenatorias ha aumentado paulatinamente siendo en $201359.68 \%$ y en $201870.46 \%$. El número general de denuncias ha aumentado de forma exponencial con 124893 en 2013 y 166961 en 2018 así como las denuncias por terceras personas (2395 en 2013; 7921 en 2018). En el año 2013 el 1.2\% de los españoles consideraba la violencia de género entre los tres principales problemas de España, mientras que el porcentaje en enero de 2019 ascendió al $7 \%$. Conclusiones: Las estrategias actuales contra la violencia de género en España han conseguido un aumento ostensible de la sensibilización de la población ante el problema y un aumento del número de denuncias y de sentencias condenatorias, sin embargo, el número total de muertes por violencia machista ha disminuido levemente por lo que es importante insistir en mejorar las estrategias existentes.

Formación Por Competencias: Valoración De Satisfacción En Cirugía De Trauma. Adrian Terán, Cristopher Varela, Adalid Gonzalez, Marco S Venturoni, Jesus, Pablo Ottolino, Fernando Rey, Juan L Rosas. From the Hospital General del Este “Dr Domingo Luciani”, Caracas, Dc, Venezuela.

Introducción: Existe la necesidad de realizar una revisión curricular del contenido referente a Cirugía de Trauma establecido en el programa de especialización en Cirugía General de la UCV por expertos en Cirugía General y de Trauma, actualizando según normativa nacional e internacional su contenido, unificando estrategias para mejorar la instrucción impartida en cada centro hospitalario. Objetivo: Valorar el nivel de satisfacción en formación por competencias en Cirugía de Trauma que poseen los residentes del último año (tercer año) del programa de especialización en Cirugía General de la Universidad central de venezuela (UCV). Materiales y métodos: Estudio de tipo prospectivo, transversal, no experimental, descriptivo. Se entrevistaron 39 residentes del tercer año de los hospitales: Universitario de Caracas, Domingo Luciani, Miguel Pérez Carreño, Vargas de Caracas, General del Oeste y Militar Carlos Arvelo. Se utilizó el método de análisis funcional para diseñar el instrumento aplicado con su respectiva validación. Para la confiabilidad se utilizó cálculo de aciertos y desaciertos de Haynes. La técnica de análisis fue estadística descriptiva. Resultados: $69 \%$ de los residentes considera su nivel de entrenamiento actual en trauma acorde a sus necesidades, siendo instruidos con clases, seminarios y conferencias en $77 \%$ de los hospitales estudiados, 59\% mediante intervenciones quirúrgicas con tutores calificados y $15 \%$ con prácticas en simuladores; $50 \%$ de los encuestados no sienten la confianza suficiente para enseñar a un residente junior en la ejecución de maniobras básicas para la atención del politraumatizado y $51 \%$ manifiesta poca satisfacción con las competencias desarrolladas. Conclusiones: la instrucción en Cirugía de Trauma para residentes del último año del curso de especialización en Cirugía General de la UCV no presenta un nivel óptimo de eficiencia en cuanto a formación didáctica, destrezas y habilidades quirúrgicas desarrolladas en la mayoría de sus centros hospitalarios, reflejadas en la satisfacción en formación por competencias en Cirugía de Trauma.

Politraumatismo Grave En Paciente Gestante Con Compromiso De Pelvis Y Reja Costal. Reporte De Un Caso. Paula AJ Muñoz, Carlos AD López, David AM Toro, Alejandro G Chávez, Daniela Carolina, Carolina R Herrera. From the Universidad de Antioquia, Antioquia/ Medellín, Colombia.

Introducción: El 7\% de las mujeres embarazadas sufren de algún tipo de traumatismo durante el embarazo, de estos la tercera parte están relacionados con accidentes de tránsito. Cuando el trauma pone en riesgo la vida materna está asociado con un 50\% de pérdida fetal. Las fracturas costales alteran la fisiología ventilatoria, lo que agregaría morbimortalidad a esta población especial. Materiales y métodos: Analizar la evolución y desenlace de una paciente gestante con trauma pélvico y de pared torácica por medio de un reporte de caso, correlación clínico-radiológica y revisión de la literatura. Resultados: Paciente de 24 años, secundigestante de 26 semanas. Accidente de tránsito en calidad de copiloto con avulsión de tejidos blandos del periné, sangrado vaginal, pelvis inestable y fracturas costales con marcado distrés respiratorio. Presenta muerte fetal por lo que realizan cesárea. En la UCI requiere intubación por lo que se considera fijación de la reja costal con lo cual presenta marcada mejoría. Fijación de la pelvis con tutor externo. Requiere realización de colostomía por contaminación de la región perineal y osteomielitis de los huesos pélvicos. Mejoría progresiva, control del foco infeccioso y alta al día 19 de hospitalización. Conclusiones: Algunos autores postulan que la sobrevida de las mujeres gestantes con traumatismos es mayor al compararlas con aquellas que no lo están en relación a su mayor reserva fisiológica y el postulado de una mejor perfusión de los órganos que las hace más tolerantes al estado de shock. El trauma de reja costal empeora la situación al realizar un cambio drástico en el proceso de oxigenación. La fijación quirúrgica ha demostrado ser una estrategia eficaz para normalizar la dinámica ventilatoria.

Dimensiones De La Desigualdad Que Influencian La Violencia Basada En Género En Colombia Durante El Periodo 2012-2016. Monica AM Garcia, Juan CR Garcia, Byran SU Trochez. From the Universidad Del Valle, Cali, Colombia.

Introducción: En los últimos veinte años, varios estudios e iniciativas en investigación han estudiado la magnitud de la violencia, los factores de riesgo asociados, la victimización y los agresores en Colombia. Por otro lado, las investigaciones que explican las causas de las desigualdades sociales presentes en la violencia basada en género son pocas, y más limitadas aun usando la metodología para medición de desigualdades 
de la OMS. El propósito de esta investigación fue analizar los patrones de las desigualdades social que influencian la violencia basada en género en Colombia durante el período 2012-2016. Materiales y métodos: Se realizó un estudio ecológico retrospectivo de todos los casos notificados en el Sistema de Vigilancia en Salud Pública SIVIGILA en el periodo 2012 a 2016. Se utilizaron como estratificadores de desigualdad: la educación, empleo, área de residencia, Índice de Necesidades Básicas Insatisfechas (NBI), Índice de Pobreza Muldimensional (IPM) y barreras de acceso a los servicios de salud. La medición de las desigualdades absolutas y relativas se estimaron a partir del Índice de Desigualdad de la Pendiente (IDP), el Índice Relativo de Desigualdad (IRD) y el Índice de Concentración. Finalmente, todos los resultados fueron analizados de forma georreferenciada. Resultados: Las NBI, barreras de acceso y el desempleo son las dimensiones que más aportaron a la explicación de la violencia basada en género. Adicionalmente, se identificaron dos patrones de desigualdad: exclusión social y desigualdad incremental, es decir, que los departamentos con mejores condiciones para estas dimensiones presentaron altas tasas de violencia basada en género. Conclusiones: Se recomiendan cuatro áreas de acción para ayudar con la reducción de las desigualdades sociales: (i) mejorar la desagregación de los datos; (ii) implementar políticas que actúen sobre los determinantes sociales y la interseccionalidad de éstos determinantes; (iii) garantizar programas de prevención de la violencia basados en la evidencia; (iv) garantizar programas con enfoque de equidad.

Estabilización Quirúrgica De La Pared Torácica En Trauma Severo De Tórax:Tres Años De Experiencia. Sergio Rivera, Roberto Margos, Rember Rosales, Luis Aragón. From the Instituto Guatemalteco de Seguridad Social.

Introducción: De los traumas de tórax, el 10\% son tórax inestables, asociados a alta mortalidad (10-36\%) 7. Menos del 1\% de los pacientes con tórax inestables son intervenidos quirúrgicamente (idealmente en menos de 72 horas post trauma). La fijación costal mejora la biomecánica del tórax, se realiza con placas de titanio anteriores. Materiales y métodos: Estudio transversal descriptivo sobre instrumentación quirúrgica realizada a siete pacientes con trauma severo de tórax en el periodo de Mayo de 2016 a Marzo de 2019 en el Hospital General de Accidentes "Ceibal" del Instituto Guatemalteco de Seguridad Social. Resultados: Siete de los 208 pacientes con trauma severo de tórax fueron instrumentados (3.36\%). La edad promedio de los pacientes fue de 40.7 años, el 100\% fueron masculinos. El $85.7 \%$ tenia una o más lesiones asociadas, predominando el trauma craneoencefálico (71.43\%). Los pacientes con trauma craneoencefálico tienen 1.5 veces más riesgo de ventilación mecánica, evidenciando además aumento de la estancia hospitalaria en 5.6 días y aumento de la ventilación mecánica en 6.5 días comparados con pacientes sin trauma craneoencefálico. La estancia hospitalaria fue de 26 días. La estancia en cuidado crítico fue de 11 días. El 57\% ameritó ventilación mecánica, durando en promedio 11 días ventilados. No hubo reintervenciones, hubo una complicación (Infección de herida operatoria), y una mortalidad atribuida a trauma craneoencefálico. El tiempo de retorno a labores fue de 88 días post trauma. Actualmente los pacientes están sin dolor, laborando en el mismo puesto y no tienen restricción física.
Conclusiones: En trauma severo de tórax, es importante determinar si el paciente es candidato para estabilización quirúrgica. El riesgo de ventilación mecánica en pacientes con trauma severo de tórax que tienen asociado trauma craneoencefálico aumenta en 1.5 veces. La estabilización quirúrgica del tórax disminuye los días de ventilación mecánica, la estancia en cuidado crítico, la mortalidad y mejora el pronóstico.

Nuevo Método Con La Utilización Del Doppler Transcraneal En La Valoración De Síndrome Compartimental De Cuello En Los Pacientes Quemados.Julio Cabrera, Corina Puppo, Leandro Moraes. From the Centro Nacional de Quemados, Montevideo, Uruguay.

Introducción: Las quemaduras circunferenciales totales o parciales de cuello pueden conducir a un síndrome compartimental en grandes quemados. El incremento de la presión intracompartimental produce colapso del sistema vascular que conduce al desarrollo de edema encefálico. Objetivo: Mostrar la utilidad del Doppler transcraneal (DTC) en el diagnóstico y evaluación de la eventualidad de un síndrome compartimental de cuello. Materiales y métodos: Se analizaron 2 pacientes, internados en el Centro Nacional de Quemados de Uruguay, que tenían quemaduras sobre cuello. Paciente No 1, N A, 57 años, femenino, peso $99 \mathrm{Kg}$. Injuria por fuego directo. Superficie corporal quemada (SCQ) profunda 58\%, quemaduras circunferenciales de los 4 miembros, tórax, cara y cuello no circunferencial. Paciente No 2, A A, 30 años, femenino, epiléptica, Peso $66 \mathrm{Kg}$. Injuria fuego directo, SCQ 14\%, de grado profunda ,circunferencial en cuello. Resultados: En el paciente No 1: Al finalizar la etapa de reanimación, $20 \mathrm{~kg}$ de incremento del peso y de la tensión del cuello. Doppler Transcraneal Arteria cerebral media (ACM) izquierda y derecha = Velocidad Media (VM) 39-cm/seg, Índice de Pulstilidad (IP) = 1,59. DTC post - escarotomía de cuello: ACM izquierda y derecha: VM $80-90 \mathrm{~cm} / \mathrm{seg}, \mathrm{IP}=0,90-1$. En el paciente No 2: Incremento del peso de $4 \mathrm{~kg}$ post-reanimación. DTC: ACM izq y der : VM 32-38 $\mathrm{cm} / \mathrm{seg}, \mathrm{IP}=1,2$. DTC post escarotomía ACM: VM $82 \mathrm{~cm} / \mathrm{seg}, \mathrm{IP}: 1$ Conclusiones: Desarrollo de síndrome compartimental de cuello, con patrón hemodinámico de alta resistencias y de hipoflujo cerebral. Este patrón se observó en quemadura no circunferencial de cuello, que requirieron grandes aportes hidroelectrolíticas en el primer caso y en el segundo en quemadura circunferencial con poco aporte DTC:Técnica de diagnóstico precoz , no invasiva, de la hipoperfusión cerebral y de control de su terapéutica.

Factores Pronosticos Para Perdida De Extremidad (Amputación), En Paciente Con Trauma Arterial Periférico (Trauma Severo De Extremidades) Atendidos En Hospital De lii Nivel - HuilaColombia 2014-2018. Conrado Jimenez, Carlos Martinez, Wilmer Botache, Rolando Medina, Edwin, Juan Sanjuan, Jhon Salamanca, Adriana Morales, Alejandra Duran. From the Universidad Surcolombiana - Hospital Universitario NeivaColombia, Neiva, Colombia.

Introducción: El trauma vascular es un evento de baja frecuencia, con una alta morbimortalidad que afecta principalmente hombres jóvenes; generalmente requiere manejo quirúrgico. Éste se asocia con múltiples complicaciones; desde la re intervención quirúrgica hasta la amputación de la extremidad; las cuales, estan influenciadas por variables asociadas al trauma directo y la atención hospitalaria. 
Materiales y métodos: Estudio observacional, analitico de corte retrospectiva con población a conveniencia de pacientes mayores de 13 años, con trauma arterial periférico, quienes recibieron manejo quirurgico en Hospital de III nivel - Colombia. Resultados: Incluye 102 pacientes con TAP; incidencia del $1,56 \%$ entre 2014 y 2018 . El $89 \%$ fueron hombres con edad promedio de 28,5 años, a su ingreso con TAM de $76,1 \mathrm{~mm} \mathrm{Hg}$ (30-136 mm Hg), un Injury severity Score (ISS) 16,63 (4-41), hemoglobina y $\mathrm{pH}$ de $9,5 \mathrm{gr} / \mathrm{dL}, 7,28$ respectivamente. La principal comorbilidad fue la farmacodependencia 8,8\%; $63 \%$ de los pacientes provenian de un radio < a $50 \mathrm{~km}$ del hospital de referencia. EI MESS (Mangled extremity severity) promedio 5,27 puntos y un tiempo critico de isquemia de extremidad $>$ a 6 horas en el 38\%. Principal compromiso de miembros superiores, secundario a heridas por arma cortopunzante. La lesion mas frecuente fue la traseccion arterial en $63 \%$ de los paciente. Dentro de las complicaciones posquirurgicas, se destacaron la trombosis del vaso $(21,5 \%)$ y la amputacion en el $13,9 \%$. Como factores de riesgo asociados a amputacion se determino, la edad $>20$ años, estancia hospitalaria $>7$ dias, MESS $>7$ puntos, tiempo de isquemia $>6$ horas y la reintervension quirugica $(p)$. Conclusiones: El trauma arterial periferico, es una patologia con gran repercusion socioeconomica en la poblacion joven, responsable de alta tasa de secuelas funcionales. Es necesario la atencion oportuna y eficaz de esta patologia, con el objetivo de influir directamente en los multiples factores de riesgo asociados a perdida de extremidad, esto con el fin de mejorar el pronostico de esta poblacion.

Tempo De Permanência Na Sala De Emergência Das Vítimas De Acidentes De Trânsito. Tatiana FC Do Nascimento, Iveth Y Whitaker, Nathalia Z De Moura, Rosimey R Thomaz, Maria Carolina. From the UNIFESP-Universidade Federal de São Paulo, São Paulo, Brazil.

Introducción: verificar o tempo de permanência na sala de emergência (SE) até o encaminhamento ao centro cirúrgico ou unidades de internação e verificar a associação do tempo na SE com as variáveis idade, sexo, relacionadas ao acidente de trânsito, ao atendimento na SE, gravidade da lesão e do trauma, relacionadas a hospitalização e pós alta. Materiales y métodos: Estudo observacional do tipo coorte prospectiva para seguimento de pacientes de acidente de trânsito, desde a admissão no pronto-socorro até seis meses após alta hospitalar. Os dados de pacientes atendidos na SE que aceitaram participar do estudo foram inseridos na plataforma Research Electronic Data Capture. Para verificar a associação entre tempo de permanência na SE e as variáveis do estudo, utilizou-se o teste de Kruskal-Wallis, teste de Qui-quadrado ou a Razão de Verossimilhança, considerando o nível de significância de $5 \%$ e intervalo de confiança de $95 \%$. Resultados: A amostra com 327 pacientes revelou média do tempo de permanência na SE de 4,8 horas. O tempo na SE mais longo associou-se ao tempo para execução da TC de crânio $(p=0,0039)$, gravidade do trauma (anatômica $p=0,0056$ e fisiológica $p=0,0141$ ) e complicações $(p=0,0241)$. Entre os que permaneceram na SE até 2 horas, o maior percentual $(37,1 \%)$ foi encaminhado ao setor Verde (p). Conclusiones: O tempo de permanência na SE associou-se aos fatores indicativos de gravidade do trauma. Nos intervalos mais demorados, foram observados pacientes de maior gravidade; nos menos demorados, os de menor gravidade. Considerando a elevada demanda de pacientes nos serviços de emergência e para que as necessidades terapêuticas e de monitorização do paciente grave possam ser atendidas em momento e local apropriado às suas condições, a organização do fluxo de pacientes hospitalizados deve compor a agenda da gestão pública dos serviços de saúde.

Curso De Mejora De Calidad En Trauma. Experiencia Argentina. Carlos Tenaillon, Maria L Ruffa, Ezequiel Monteverde, Marilina Santero, Laura, Jorge Neira. From the Fundación Trauma, Buenos Aires, Argentina.

Introducción: La capacitación del equipo de salud es un aspecto fundamental del Programa de Fundación Trauma (FT), que desde 2009 brinda cursos con respaldo científico a las instituciones de la Red de Trauma de la Provincia de Buenos Aires. Describir y evaluar el curso Mejora de la Calidad en Trauma (MCT), que se inició en 2016 con la formación de instructores locales. En Argentina es el primer curso dirigido al monitoreo de los servicios que atienden trauma y es un componente clave para del programa de trabajo de FT. Materiales y métodos: Análisis descriptivo de los cursos realizados entre abril 2016 y marzo 2019, a partir de datos del Registro de Capacitación de FT y de una encuesta online post-curso implementada desde agosto de 2018. Resultados: Se realizaron 8 cursos donde asistieron 165 alumnos (64.2\% mujeres, $n=106)$ con una edad (mediana, RIC) de 44 años (37-50); 70.9\% fueron médicos, $13.3 \%$ enfermeros, $10.9 \%$ administrativos y $4.8 \%$ otros. Dentro de los médicos, las especialidades fueron terapia intensiva, cirugía y pediatría $(20.3 \%$, $19 \%$ y $17.7 \%$, respectivamente). El $86.7 \%(n=143)$ pertenecieron a hospitales, $6.7 \%(n=11)$ a sistemas prehospitalarios. Sólo el $14.5 \%$ $(n=24)$ de los alumnos abonaron el curso, el $85.5 \%(n=141)$ fueron becados por FT. La encuesta se administró a 34 participantes, con una tasa de respuesta de $59 \%(n=20)$, el $100 \%$ consideró los contenidos buenos o muy buenos, el $65 \%$ que podría aplicar los conocimientos adquiridos y el $70 \%$ que recomendaría el curso. Conclusiones: Las estrategias de MCT son un componente insustituible de los programas de trauma y deberían ser adoptadas por todas las instituciones que atiendan lesionados para minimizar la brecha entre la teoría y la práctica. En Argentina, FT propone el curso de MCT para que los profesionales puedan implementar distintas estrategias, incluyendo análisis cuantitativos de resultados y técnicas avanzadas de identificación de la mortalidad prevenible.

Trauma En Niños vs Adultos: Análisis Del Registro De Trauma De Fundación Trauma, Buenos Aires Argentina. Ezequiel Monteverde, Marilina Santero, Laura Bosque, Jorge Neira. From the Fundación Trauma, Buenos Aires, Argentina.

Introducción: El traumatismo encefalocraneano (TEC) es de las condiciones clínicas más frecuentes en trauma y constituye un importante problema de salud pública tanto en Argentina como en el mundo, con diferencias en la evolución post-TEC entre niños y adultos. Nuestro objetivo es analizar características epidemiológicas y evolución en pacientes con TEC según edad. Materiales y métodos: Análisis retrospectivo observacional de ingresos prospectivos al Registro de Trauma de Fundación Trauma en 10 hospitales entre 2010-2016.Criterios de inclusión:AIS-3 en cabeza yAIS-2enotras regiones.Lamuestra sedividióen:0-17,18-55y > 55años. Resultados: Se analizaron 1.275 adultos y 280 niños. Las causas fueron (adultos vs niños): motociclista (45.2\% vs $28.9 \%$ ), agresiones 
(12.4\% vs $8.2 \%$ ) y caídas (12.1\% vs $15.3 \%$ ). Los adultos tuvieron más compromiso neurológico inicial (Glasgow-8: $33.3 \%$ vs $21.1 \%$ ) y mayor severidad: ISS-16: $57.2 \%$ vs $42.9 \%$ y NISS-16: $84.4 \%$ vs $72.1 \%$ (p). Conclusiones: Los niños muestran menos lesiones asociadas al TEC y menor severidad global. El uso de recursos y la incidencia de complicaciones fueron similares. Los

Índice De Trauma vs Triss Para Predicción De Mortalidad En Pacientes Traumatizados. Ezequiel Monteverde, Santiago Donikian, Marilina Santero, Laura Bosque, Jorge. From the Fundación Trauma, Buenos Aires, Argentina.

Introducción: La clasificación correcta de los pacientes traumatizados resulta fundamental para la evaluación de resultados según estándares de atención. Aunque el sistema de clasificación de referencia es el TRISS (Trauma and Injury Severity Score) una barrera para usarlo es contar con todas las variables requeridas para su cálculo. El objetivo de este estudio es comparar la performance en la predicción de mortalidad según método TRISS y un algoritmo desarrollado por Fundación Trauma. Materiales y métodos: Fundación Trauma desarrolló en Argentina un Programa de Trauma que se encuentra activo en 14 hospitales de la provincia de Buenos Aires, cuya pieza fundamental es el Registro de Trauma. En base a 23 variables del Registro se desarrolló un modelo predictivo de mortalidad usando el paquete xgboost en R. El modelo se comparó con TRISS en relación a calibración (Hosmer-Lemeshow, HL), discriminación (curva ROC) y precisión. Las áreas bajo la curva $(A B C)$ se calcularon por el método De Long. Se reportan intervalos de confianza de 95\% (IC95\%). Resultados: El modelo (índice de trauma, IT) se desarrolló usando datos de 12.640 casos de pacientes mayores de 17 años y se testeó en 33.09. El test de HL mostró que ninguno de los modelos logró una adecuada calibración (ambos p). Conclusiones: Cuando se dispone de un registro de trauma basado en estándares internacionales y adecuado a la realidad local, la performance de modelos predictivos basados en algoritmos es al menos tan buena como la de los modelos de referencia, con el beneficio de poder usarse en ausencia de algunos datos clave.

Trauma Y Salud Pública: Impacto En Años De Vida Ajustados Por Discapacidad. Análisis Del Registro De Trauma De Fundación Trauma, Buenos Aires Argentina. Ezequiel Monteverde, Marilina Santero, Laura Bosque, Jorge Neira. From the Fundación Trauma, Buenos Aires, Argentina.

Introducción: Los años de vida ajustados por discapacidad (AVAD o DALY) miden el impacto de enfermedades en la salud pública. Un AVAD equivale a un año de vida saludable perdido por enfermedad, discapacidad o muerte prematura. El objetivo del estudio es estimar carga de enfermedad y evaluar impacto de la enfermedad trauma, calculando los AVAD asociados. Materiales y métodos: Análisis de ingresos prospectivos al Registro de Trauma de Fundación Trauma en 14 hospitales, de la provincia de Buenos Aires, entre 2010-2019. Para el cálculo de los AVAD se estimaron los años de vida perdidos (AVP), diferencia entre esperanza de vida y edad del deceso, y años vividos con discapacidad (AVD) desde el trauma y ponderados por la discapacidad. Estas 2 estimaciones se suman para obtener los AVAD.
La ponderación por discapacidad se calculó por el diagnóstico principal por CIE10 (capítulo XIX) y los mecanismos lesionales se clasificaron por CIE10 (capítulo XX), reagrupándose en 9 clases. Resultados: Se analizaron 29.728 hechos (71.4\% varones). La mediana de edad en mujeres fue 19 (rango intercuartil (RIC) 7-43) y en varones, 24 (RIC: 14-38). El mecanismo más frecuente fue lesiones por transporte (31.9\%), luego caídas (23\%) y violencia interpersonal (15.5\%). En total 495.173 AVAD, compuestos por 52.281 AVP y 442.892 AVD. El mayor número de AVAD correspondió a lesiones por transporte (163.525 AVAD, compuestos por 23.964 AVP y 139.561 AVD). Cuando se normalizaron por la cantidad de hechos en cada clase, el mayor impacto en AVP correspondió a ahogamiento (5.4 años/hecho) y violencia interpersonal (3.1 años/hecho) y en AVD a ahogamiento (54.6 años/hecho) e intoxicaciones (17.9 años/hecho). Conclusiones: El cálculo de los AVAD permite estimar el impacto real del trauma, que se oculta si sólo se reporta mortalidad. En esta investigación se comprobó que por cada año de vida perdido por las muertes del trauma se pierden 8.5 por discapacidad.

Depuración De Lactato En Trauma a Gran Altitud. José AV Soto, Jorge Jiris, Jorge Antezana, Felix Caceres. From the Centro De Trauma Hospital Corazón De Jesús, El Alto, Bolivia.

Introducción: La resucitación cuantitativa en pacientes críticamente enfermos comprende intervenciones hemodinámicas tales como la expansión del volumen intravascular y soporte vasoactivo buscando alcanzar parámetros fisiológicos en el menor tiempo posible. El estudio pretende caracterizar la depuración de lactato en pacientes de trauma críticamente enfermos, en la Unidad de Cuidados Intensivos de un Centro de Trauma a gran altitud. Materiales y métodos: Estudio retrospectivo transversal observacional. Unidad de Cuidados Intensivos de Adultos del "Centro de Trauma-Hospital Corazón de Jesús" de la ciudad de El Alto, La Paz (Bolivia), a 4090 metros sobre el nivel del mar, periodo 01 de marzo 2017 al 31 de marzo 2019. Se incluyeron todos los pacientes de Trauma ingresados a la Unidad de Cuidados Intensivos de Adultos, nativos de gran altitud así como residentes de la misma por lo menos los últimos 6 meses. Resultados: Se incluyeron 160 pacientes, con sobrevida de 85\%, promedio de edad 44 años; en el grupo de 136 supervivientes, alrededor de la mitad del lactato de ingreso fue depurado (49.9\%), a comparación del grupo de 24 fallecidos, en el cual incluso el valor de depuración de lactato fue menor a la décima parte (8\%), ambos con $p-0.05$ mediante la prueba de t de Student. Conclusiones: Una adecuada depuración de lactato en pacientes de trauma críticamente enfermos nativos y residentes de gran altitud, se asocia a mayor supervivencia y debería ser considerado como un objetivo durante la reanimación en pacientes críticos, de forma similar a estudios realizados en otras latitudes.

Motociclistas Com Lesões Traumáticas Decorrentes De Acidente De Trânsito: Análise Da Morbidade E Mortalidade. Rosimey $R$ Thomaz, Iveth $Y$ Whitaker, Caroline De Lima Neto Silva, Tatiana FC Do Nascimento Nascimento, Nathalia Z De Moura, Maria CB Teixeira Lopes, Giane L Araujo. From the Universidade Federal De São Paulo, São Paulo, Brazil.

Introducción: Os acidentes de trânsito constituem um problema de saúde pública já que causam grande número de óbitos no 
mundo e no Brasil. Objetivos: verificar fatores relacionados ao paciente, ao evento, à hospitalização, a recuperação pós alta e à gravidade do trauma associados a mortalidade e identificar fatores de risco independentes associados à mortalidade em motociclistas com lesões traumáticas decorrentes de acidente de trânsito. Materiales y métodos: Coorte prospectiva com seguimento de motociclistas hospitalizados, desde a sua admissão no pronto socorro até seis meses após a alta ou óbito hospitalar. A amostra incluiu motociclistas envolvidos em acidentes de trânsito e hospitalizados. Os testes Exato de Fisher ou Razão de Verossimilhança, Mann-Whitney e regressão logística foram aplicados, considerando o nível de significância de $5 \%$. Resultados: De 190 motociclista, 93,8\% eram do sexo masculino com média de idade de 30,1 anos, em sua maioria condutores (88,9\%) e $16,4 \%$ estavam sem capacete. As lesões mais frequentes foram em membros inferiores (66,3\%). A média de internação foi de 8,6 dias, $16,8 \%$ tiveram complicações, a mortalidade foi de $5,3 \%$ e a boa recuperação e total foi de $48,8 \%$. As chances de óbito foram mais elevadas nos motociclistas sem capacete $(O R=5,8)$ e com complicações (OR $=147,9)$. A redução no escore da Escala de Coma de Glasgow aumentou em $73 \%$ e $66 \%$ a mortalidade no atendimento pré-hospitalar e na sala de emergência. As chances de óbito aumentaram em $8 \%$ a cada dia de internação e em $25 \%$ com aumento da gravidade do trauma, sendo este um fator de risco independente associado à mortalidade. Conclusiones: Na amostra estudada houve predomínio de jovens do sexo masculino com lesões traumáticas principalmente nas extremidades. Escores ECGI baixos, ocorrência de complicações, tempo de internação e ausência de capacete associaram-se ao óbito. A gravidade do trauma foi identificada como fator de risco independente para mortalidade.

Mortality in Traumatic Rupture of the Aorta; Mechanism Doesn't Matter. Faran Bokhari, Chih-Yuan Fu, Francesco Bajani, Francesca Bryan, Marissa, Frederic Starr, Andrew Dennis, Matthew Kaminsky, Victoria Schlanser, Stathis Poulakidas. From the Cook County Hospital, Chicago, USA.

Introduction: Traumatic rupture of the aorta (TRA) is rare and not well described in falls. The aim of this study was to establish the risks and outcomes of thoracic aortic rupture in falls vs motor vehicle accidents (MVAs). All factors that might influence outcomes in either mechanism for TRA were analyzed. Materials and methods: The National Trauma Data Bank was queried for thoracic aortic injuries treated at all trauma centers from July 1, 2013 to June 30,2015 . A retrospective analysis was performed to define the effects of age, SBP, protective devices, gender, and ISS on TRA incidence and mortality. Results: 106,227 MVA patients and 33,244 falls were analyzed. 2,655 (1.7\%) MVAs and $160(0.5 \%)$ of falls incurred thoracic aortic injuries. Patients with TRA from MVA or falls were not significantly different in age, sex, ISS, SBP, and incidence of TRA (Age (41.4 vs 43.9 years, $p=0.156)$, male (70\% vs $76.9 \% p=$ $0.096)$, ISS (38.9 vs $37.4, p=0.258)$, SBP (91.8 vs $86.6 p=0.243$ ) and mortality $(15.4 \%$ vs $18.8 \% p=0.257)$ ). The rate of TRA with protective devices in MVA was $32 \%$ vs $75 \% p<0.001$. For falls with protective devices the rate of TRA was $11.3 \%$ vs $14.4 \% p<0.305$. Conclusion: Thoracic aortic injury is a rare condition with high mortality. Although MVAs cause thoracic aortic injury at a rate
5 times that of falls, the high mortality rates are not significantly different. While protective devices are effective in protecting against TRA in MVAs, our study does not indicate a similar protective effect in falls.

Examining the Surgical System in Amazonas, Brazil Using a Hospital Assessment Tool. Jessica Correia, José E Souza, Pedro H Gomes, Rodrigo Vaz, Saurabh, Isabelle Citron, Lina Roa, Paul Truche, Cleinaldo Costa, Nivaldo Alonso. From the UEA, Amazonas/Manaus, Brazil.

Introduction: Surgical infrastructure remains understudied in the remote areas of the Amazon. A quantitative hospital assessment tool (HAT), previously developed to assess surgical infrastructure, services and workforce at a hospital level, is being deployed to assess surgical care in Brazil's largest and most rural state, Amazonas and identify potential areas of improvement. Materials and methods: A randomized stratified selection of hospitals across Amazonas, that reported having performed surgical procedures in 2015, was performed. Hospitals were stratified by population quartile and selected at random within each stratum. A formal surgical infrastructure assessment was performed using the HAT at each site through quantitative surveys, and surgical log book review. Results: The HAT was deployed at 18 hospitals. Half of the hospitals have no internet access, but $95 \%$ have consistent power. None of the hospitals had an intensive care unit. In three of them, no operating rooms were found operative. No hospital reported use of the WHO safe surgery checklist. Inhalation general anesthesia is available at $3 / 18$ hospitals; IV sedation $15 / 18$, spinal and regional anesthesia is available at the $17 / 18$ hospitals. Blood bank services are available at $16 / 18$ hospitals. The surgeons, anesthesiologists and obstetricians density was estimated at 7.63 per 100,000 inhabitants. $72.8 \%$ of the hospitals do not have an anesthesiologist available $24 / 7,72.2 \%$ do not have an OBGYN available $24 / 7$ and at $77.8 \%$ there are no scrub nurses or technicians on staff. Only $33 \%$ of municipalities report 2 hour access to surgical care more than $75 \%$ of the time. Conclusion: Preliminary results show a significant lack of availability of surgical staff and lack of basic tools to perform safe surgery across the rural municipalities of Amazonas. This project provides the framework for a successful partnership engaging local stakeholders to take part in meaningful research to evaluate and influence regional surgical agendas.

Injerto De Peritoneo Para Corrección De Lesion De Vena Iliaca: Un Reporte De Caso. Manuel Giraldo, Michael Zapata, Adolfo Gonzalez. From the Hospital Universitario Del Valle, Valle Del Cauca/Cali, Colombia.

Introducción: Las lesiones vasculares por trauma estan asociadas a una gran morbi-mortalidad, en la mayoria de los casos las lesiones destructivas de grandes venas son manejadas con ligadura o con rafias que estrechan la luz del vaso generando complicaciones como trombosis y/o edema cronico, que compromete la calidad de vida del paciente. Materiales y métodos: Describir el caso de un paciente a quien se le realiza injerto de peritoneo para corrección de lesión vascular venosa mayor. Reporte de caso, de un paciente con lesion de la vena iliaca externa izquierda reparada con parche de peritoneo. Resultados: Paciente de 18 años, que ingresa con herida unica por arma de fuego en glúteo derecho, con abdomen agudo y tacto 
rectal positivo; es llevado a laparotomia exploratoria encontrándo: hemoperitoneo 2000 cc, herida de ileon, vejiga, recto extraperitoneal y una lesión en vena iliaca externa de $3 \mathrm{~cm}$ de longitud y $70 \%$ de su circunferencia. La lesión venosa se corrige con injerto de peritoneo obtenido de cara posterior de recto abdominal, se realiza colostomía de sigmoides y terapia VAC. Posteriormente es llevado a cierre de cavidad. Durante posoperatorio se realiza ecografia doppler de miembro inferior que muestra flujo adecuado, sin signos de trombosis ni edema de MII. Finalmente se da egreso por buena evolución clinica. Conclusiones: En las lesiones venosas destructivas de miembros inferiores, generalmente se opta por realizar ligadura, en este caso se recurrio a un injerto de peritoneo, exitoso sin trombosis ni edema. Este es el primer reporte de casos de una lesion de iliaca externa manejada con pache de peritoneo.

Caracterización Demográfica Y Clínica DelTrauma Vascular Periférico En El Hospital Universitario Del Caribe, Cartagena, Colombia, Entre 2015 Y 2017. Vanesa L Ramos, Jaime D De Hoyos, Ana S Arrieta, Laura J Santamaría, Felipe, Juan D Maya, Efraín R Barakat. From the Universidad de Cartagena, Cartagena, Colombia.

Introducción: En trauma, las lesiones vasculares tienen gran morbilidad y mortalidad, principalmente a nivel toracoabdominal. El trauma vascular periférico (TVP) es aquel que comprende tanto las lesiones de las ramas axilobraquiales de las extremidades superiores, como las femoropoplíteas en las extremidades inferiores; tiene una incidencia baja, con principal afección a personas en edad productiva y que con lleva un impacto en años de vida potencialmente perdidos. Materiales y métodos: Estudio observacional descriptivo retrospectivo longitudinal de los pacientes con trauma vascular periférico con requerimiento de intervención quirúrgica en la E.S.E. Hospital Universitario del Caribe entre enero de 2015 y diciembre de 2017, que incluye variables sociodemográficas, relacionadas con el trauma, clínicas, relacionadas con el manejo quirúrgico y complicaciones. Resultados: Se encontraron 87 pacientes, de género masculino en $92 \%$. El principal mecanismo de lesión fue penetrante por arma cortopunzante en $80,5 \%$. Los principales signos duros fueron el sangrado pulsátil en $62,1 \%$ y la ausencia de pulsos en $46 \%$. La distribución del trauma vascular fue principalmente en miembros superiores, arteria cubital, radial y braquial respectivamente. La técnica quirúrgica que se presentó con mayor frecuencia fue la ligadura en $56,3 \%$, luego la anastomosis $31 \%$. La mediana de estancia hospitalaria fue 3 días. La frecuencia de amputaciones fue de 1,2\% y la mortalidad 3,5\%. Los pacientes con MESS $>7$ y requerimiento de transfusiones tuvieron mayor necesidad de ingreso a $\mathrm{UCl}$, estancia hospitalaria y presencia de shock hipovolémico Conclusiones: El manejo del trauma vascular periférico es un reto para el cirujano general y debe ser individualizado con el uso de las herramientas necesarias para la preservación de la extremidad.

Comportamiento En La Atención Prehospitalaria Por Parte Del Benemérito Cuerpo De Bomberos Voluntarios De Cuenca, 2014-2018. Ariel Q Gutierrez, Cesar M Almeida, Milena S Plaza, Juan CS Molina. From the Benemérito Cuerpo de Bomberos Voluntarios de Cuenca, Azuay/Cuenca, Ecuador.

Introducción: Durante 74 años, el Benemérito Cuerpo de Bomberos voluntarios de Cuenca (BCBVC), ha jugado un rol fundamental en la Atención prehospitalaria (APH) de pacientes con trauma, al ser la principal causa de emergencias, por lo que consideramos necesario realizar un análisis para conocer el comportamiento de las emergencias atendidas, la gravedad de los pacientes manejados en las ambulancias. Materiales y métodos: Estudio retrospectivo transversal, base de datos del BCBVC, periodo comprendido entre el 1 de enero de 2014 a diciembre de 2018, se utilizó estadística simple para su análisis y se realizaron gráficos térmicos de distribución territorial. Resultados: Periodo 2014-2018 se registraron 14.452 atenciones, con un promedio de 2.890 por año, 8 despachos efectivos día, 29\% correspondieron a accidentes de tránsito, grupo etario más afectado de 21 a 30 años con $26,6 \%$, y de 11 a 40 años $58.1 \%$, sexo masculino $61 \%$, se identificaron las parroquias con mayor accidentalidad, se realizó la resolución a nivel de APH en $12 \%$ y se trasladaron al nivel de hospitales públicos $89.5 \%$, al centro de trauma y emergencias zonal el $49.3 \%$. El trauma corresponde al $56,6 \%$ y el trauma cráneoencefálico al $13 \%$. Se identificó a Julio como el mes de mayor accidentalidad, al sábado con el mayor número de eventos. Durante la semana las horas de mayor despacho entre las 18:00 y 21:00. Conclusiones: Esta información es relevante para la monitorización del SEM de nuestra localidad, se corrobora las tendencias durante el transcurso del tiempo para la administración de recursos, fortalecer la APH por parte del BCBVC y la atención en el centro de trauma y emergencias.

Rendimiento De Escalas De Trauma En Accidentes De Tránsito En Un Hospital De Alta Complejidad Para Predecir Mortalidad. Hanier H Agudelo Ledesma, Alexei R Diaz, Laura CR Mazuera, Roberth O Martinez, Natalie. From the Universidad del Cauca, Popayan, Colombia.

Introducción: Los accidentes de tránsito en el mundo viene en incremento y cada vez más se convierte en un reto diagnostico la predicción de mortalidad con escalas anatómicas y fisiológicas de trauma. Las escalas de puntuación de trauma son la piedra angular de la epidemiologia del trauma. No existe evaluacion de rendimiento en la poblacion seleccionada Materiales y métodos: El objetivo principal de este estudio fue examinar los resultados y el rendimiento de las puntuaciones ISS, NISS Y RTS con variables subyacentes e independientes para identificar el mejor sistema de puntuación y predictor de morbilidad y mortalidad en una población expuesta. Materiales: Estudio prospectivo de corte trasversal que ingresan durante el periodo agosto de 2017 a agosto 2018 a un hospital de III de atención donde se evaluaron 650 pacientes las variables se analizaron mirar la normalidad de distribución. El rendimiento se evaluo con de las diferentes escalas, su sensibilidad, especificidad, valores predictivos, likehood ratio, Odds Ratio diagnóstico, OR de prevalencia. Resultados: La edad promedio fue de 36 años, la mayoría de accidentes de tránsito ocurrieron en hombres (70.9\%). El lugar del accidente el 59.2\% fue urban; el tipo de vehículo, un $70.8 \%$ de los accidentes fueron en motocicleta, 12.3\% peatón, de estos el $24.2 \%$ de alta energía. El $2.5 \%$ de los pacientes falleció. La sensibilidad de todas las escalas es adecuada, con mayor sensibilidad para ISS y NISS, y con mayor especificidad RTS de la misma manera el LR+ varía entre 23,7 para el RTS y 5,88 para NISS. LR-es adecuado para ISS y NISS y pobre para RTS lo cual implica un adecuado rendimiento de las escalas. Conclusiones: Las escalas de trauma ISS, NISS y RTS tuvieron un 
rendimiento adecuado en esta población a estudio con buena sensibilidad y especificidad y LR + y LR - adecuados.

An Urban Level One Trauma Center Desert Along The Us-Mexico Border: A Descriptive Analysis of Trauma Transfers From the Rio Grande Valley, Texas. Cera Kroenke, Shawn Izadi, Demba Fofana, Hansapani Rodrigo, Dawn, Ryan Shine, Kristina Vatcheva, Carlos G Cantu, Samuel Snyder, Jeffrey Skubic. From the University of Texas-Rio Grande Valley/Doctor's Hospital at Renaissance, Texas/Edinburg, US.

Introduction: The Rio Grande Valley (RGV) in Texas is one of the fastest growing urban populations in the United States (US) (currently 1.3 million documented citizens). It is one of few remaining large urban areas in the US without a level one trauma center. It is geographically isolated along the United States Mexico border, with the nearest American College of Surgeons (ACS) verified level one trauma center being 250 miles away. This study sought to describe the types and volume of trauma patients being transferred from the RGV to San Antonio, TX (SA). Materials and methods: Data on all trauma transfers from the four counties of the RGV to SA was obtained for the years 2015-2018 from the Southwest Texas Regional Advisory Council (STRAC) dataset. A retrospective cross-sectional study was performed examining demographics, types of injury, transfer method, and discharge criteria. Result: From 2015-2018, there were 836 total transfers from the RGV to SA (67\% male, 33\% female). Roughly half of the transferred population was under 18 years old. 402 (48\%) patients suffered a blunt injury, 202 (24\%) burn injuries, 81 (10\%) penetrating injuries, 145 (17\%) multiple coded injuries, and 6 (1\%) noncoded injuries. Of the patients transferred, the majority were sent via ground ambulance (70\%) with a smaller percent via helicopter/fixed-wing ambulance (25\%). 98\% of transferred patients survived to discharge from the receiving hospital. Conclusion: In the RGV, Texas over 200 trauma patients are transferred 250 miles annually to the nearest level one trauma center in SA. Further studies are needed to examine the effects of the lack of an ACS verified level one trauma center for a geographically isolated, densely populated region within the US.

Detrás Del Bisturí: La Realidad De La Cirujana Ecuatoriana. Estudio Cuali-Cuantitativo-2019. Doris AS Altamirano, Nube LF Lazo, Catherine MC Ordoñez, Sara CL Yascaribay, Ruth Marianela, Diana $K A$ Bravo. From the Universidad Del Azuay_Cedia, Cuenca, Ecuador.

Introducción: En la edad media se prohibía a mujeres practicar la cirugía a menos que asumieran las prácticas de sus maridos luego de su muerte o que un jurado las juzgara adecuadas, el objetivo fue determinar las barreras que obstaculiza a una cirujana a ocupar un lugar en la sociedad ecuatoriana. Materiales y métodos: Estudio cuali-cuantitativo, se usó frecuencias, y método etnográfico a través de entrevistas semiestructuradas y a profundidad. Muestra estratificada por provincias, se incluyó 200 (47\%) mujeres cirujanas: generales, oncólogas, urólogas, traumatólogas, pediatras, vasculares, neurocirujanas, cardiovasculares, cardiotorácicas, coloproctologas y plásticas; se envióla encuesta a 200 hombres cirujanos, 27 residentes de postgrado, 30 pacientes y 30 especialistas. Se estudio datos demográficos, académicos, percepción, discriminación, abuso sexual y laboral.
Resultados: Hay $13,71 \%$ cirujanas, la media de edad 37,69 . El $60 \%$ son madres. El 7,2\% de mujeres no ejerce. El 30,2\% de hombres y $37 \%$ de mujeres posee una maestría, PHD o subespecialidad. El $46,4 \%$ de mujeres ha ejercido un cargo administrativo. Los hombres publican más artículos y libros con diferencia estadística. La relación hombre mujer en los lugares de trabajo fue 7:1, los mentores en su mayoría fueron masculinos. La profesión ha causado problemas afectivos para mujeres y hombres. Las mujeres (63\%) han postergado o dicho no a la maternidad. Las mujeres más que hombres han rechazado propuestas laborales y académicas por atender la familia. Las horas dedicadas al hogar son mayores en mujeres con diferencia estadística. Las mujeres reciben menos apoyo de su pareja, y han sufrido mayor discriminación por parte de autoridades, compañeros o pacientes. Conclusiones: A pesar del aumento de mujeres en cirugía general y subespecialidades, campos dominados por hombres, existe aun subrepresentación y discriminación; la mujer ejerce un papel mayor en la atención del hogar y mayor sacrificio familiar y laboral para ocupar un lugar en la sociedad.

Pruebas De Laboratorio Como Predictores De Coledocolitiasis En Pacientes Sometidos a Colangiopancreatografía Retrógrada Endoscópica. Hospital José Carrasco Arteaga. Cuenca-Ecuador, 2019. Jonathan AA Yunga, Doris AS Altamirano, Raul DV Pulla. From the Hospital José Carrasco Arteaga-Universidad Del Azuay-Cedia, Cuenca, Ecuador.

Introducción: La coledocolitiasis es una complicación de la litiasis vesicular, ocurre en el $20 \%$ de casos, puede producir complicaciones como colangitis, pancreatitis y muerte. La colangiopancreatografía retrógrada endoscópica (CPRE) es una técnica directa y segura para evaluar y tratar la enfermedad pancreática y biliar. El objetivo principal del estudio fue conocer la sensibilidad, especificidad, valores predictivos e índices de verosimilitud de los parámetros de laboratorio como predictores de coledocolitiasis. Materiales y métodos: Estudio de validación de pruebas diagnósticas en 400 pacientes que presentaron síndrome Colestásico. Se estudió variables demográficas y resultados de laboratorio como gama glutamil transpeptidasa (GGT), fosfatasa alcalina (FA), amilasa, lipasa, transaminasa glutámico-pirúvica (TGP) y transaminasa glutámico-oxalacética (TGO), bilirrubinas y hallazgos en ecografía y colangioresonancia magnética nuclear (CRMN). La prueba de oro fue la CPRE. Se calculó sensibilidad, especificidad, valores predictivos, índice de versimilitud, puntos de corte y curva ROC. Resultados: la media de edad fue 60 años, el sexo femenino fue más prevalente $(54,8 \%)$. El $70 \%$ de los pacientes presentaron coledocolitiasis; la media del tamaño del cálculo fue $9 \mathrm{~mm}$; la media del diámetro del colédoco fue $11 \mathrm{~mm}$; el 6\% presentó pancreatitis, correlacionados con amilasa y lipasa; al $24 \%$ se solicitó una CRMN, con una sensibilidad y especificidad del 80 y $95 \%$ respectivamente; el $4 \%$ de la población presentó una coledocolitiasis residual o recidivante; la GGT presentó una sensibilidad $96.70 \%$, especificidad 98.98\%; la FA una sensibilidad $91.52 \%$ y especificidad $99.74 \%$; la TGO y TGP alcanzaron una sensibilidad y especificidad del $82,4 \%$ y $80,5 \%$ respectivamente. El área bajo la curva para la GGT y FA fue 0,9; es decir un excelente test para predecir coledocolitiasis. Conclusiones: Los cambios en las enzimas canaliculares y transaminasas son sugerentes para la coledocolitiasis preoperatoria; GGT mostró mejor sensibilidad y fosfatasa alcalina mayor 
especificidad; conviertiéndose en pruebas confiables como predictores de la enfermedad.

“Data Gap": Desafío Para Las Estrategias De Mejora De La Calidad En Trauma. Joaquín Baliña, Santiago Donikian, Marilina Santero, Betina Lartigue, Laura, Ezequiel Monteverde, Jorge Neira. From the Fundación Trauma, Capital Federal (Caba), Argentina.

Introducción: La implementación de los programas de mejora de la calidad en trauma requiere de datos de calidad que permitan identificar oportunidades de mejora. El objetivo de este trabajo es evaluar la brecha (missings) de información básica necesaria para la implementaciónymonitoreo delas estrategias demejora dela calidad. Materiales y métodos: Estudio retrospectivo observacional analizando la calidad de 48 campos de 4.489 hechos ingresados en el Registro de Trauma de Fundación Trauma en 13 hospitales durante el año 2018. Los datos fueron distribuidos y analizados en 6 categorías: datos del hecho; atención prehospitalaria; datos del paciente; ingreso al hospital y proceso de atención; signos vitales; comorbilidades y lesión; e índices y scores de trauma. Resultados: Al analizar los 48 campos en su conjunto, se encontró un promedio de completitud de 67\%. De 13 hospitales analizados, el que contaba con mejor calidad de los datos presentaba un promedio de completitud de $92 \%$; y el de peor calidad $58 \%$. Considerando la media de completitud, las categorías se distribuyen en el siguiente orden: (1) datos del paciente, 97.5\%; (2) índices y scores, 71.2\%; (3) hecho, 68.6\%; (4) signos vitales, comorbilidades y lesión, 66.5\%; (5) atención prehospitalaria, 62\%; (6) ingreso y proceso de la atención, $60 \%$. Conclusiones: La distribución de datos completos presenta una amplia variación entre las categorías analizadas, teniendo en un extremo los datos del paciente y lesiones, y los procedimientos y complicaciones en el otro. En términos generales, si bien se cuenta con información para trabajar en la implementación y monitoreo de estrategias de mejora de la calidad, la brecha (gap) de información se torna un desafío en sí mismo. Por ello, es necesario contar con estrategias específicas orientadas a mejorar la calidad de los datos de la fuente del Registro de Trauma: las Historias Clínicas.

Venous Thromboembolism in Geriatric Trauma Patients: Risk Factors and Associated Outcomes. Kartik Prabhakaran, Shekhar Gogna, Gary Lombardo, Ryan Malcom, Rifat. From the Westchester Medical Center, New York, USA.

Introduction: Trauma is a hypercoagulable state, and when coupled with advanced age, increases the risk of morbidity and mortality. The purpose of this study was to determine the risk factors for venous thromboembolism (VTE) in geriatric trauma patients and to study associated outcomes. We also assessed the impact of frailty, and the type and timing of VTE prophylaxis on the development of VTE. Materials and methods: Methods: We performed a 2-year retrospective review of trauma quality improvement project (ACSTQIP) database from 2014 to 2016. 381,669 patients aged -65 years were included in the study. Data on demographics, injury severity, vital signs, glasgow coma scale (GCS), type and timing of VTE prophylaxis, length of stay, and mortality were collected. Student $t$ test, ANOVA, Chi-square test and multivariable regression analyses were performed to identify independent predictors of developing VTE. Results: Of 381,669 geriatric trauma patients, 4,371 developed VTE
(1.1\%). Spine injury $(p=0.002)$, lower extremity injury $(p<0.001)$, age between 75 and 84 years $(p<0.001)$, frailty $(p<0.001)$, severe traumatic brain injury (TBI) $(p<0.001)$, duration of mechanical ventilation $(p<0.001)$ and transfusion of fresh frozen plasma (FFP) in first 24 hours of admission ( $p<0.001$ ) were independent predictors of developing VTE after trauma in elderly. Elderly who developed VTE had higher mortality $(8.4 \%$ vs $3.4 \%, p<0.001)$, hospital length of stay (18.4 vs 6.7 days, $p<0.001$ ), ICU length of stay ( 9.7 vs 1.5 days, $p<0.001)$, and increased odds of adverse discharge disposition. Conclusion: Frailty, severe TBI, spine injury, lower extremity injury, duration of mechanical ventilation, and transfusion of plasma products were independent predictors of developing VTE. The type and timing of DVT prophylaxis were not significant predictors of developing VTE. Development of VTE in geriatric trauma patients was associated with increased hospital and ICU length of stay, higher mortality, and adverse discharge disposition.

Estrategia Curricular "Ecografía en Cirugía General". Arián A Ariosa, Oscar D Pi, Hedgar B Gutiérrez, Natascha M De Pedro. From the Universidad De Ciencias Médicas De La Habana Facultad De Ciencias Médicas "Miguel Enríquez" Hospital ClínicoQuirúrgico “Miguel Enríquez", La Habana, Cuba.

Introducción: A nivel mundial el uso del ultrasonido en el departamento de urgencia se considera una herramienta importante para la evaluación inicial del paciente con patologías quirúrgicas. Un fantasma recorre el mundo, apoderándose de millones de vidas, el TRAUMA, siendo considerada la pandemia no resuelta en la era moderna. En la carrera de medicina, en nuestro país, se aborda escuetamente dicho tema, formándose profesionales de la salud con poca preparación para el diagnóstico ultrasonográfico de patologías quirúrgicas de urgencias y relacionadas con el trauma. Materiales y métodos: Se confeccionó una estrategia curricular para facilitar el proceso de enseñanza aprendizaje del ultrasonido en cirugía general, haciendo hincapié en el trauma y la cirugía de urgencia, de manera teórico-práctico. Para comprobar su eficiencia y utilidad, se realizó un estudio de desarrollo, explicativo, longitudinal prospectivo y pre experimental, con una muestra de 107 estudiantes; con el objetivo de validar la estrategia curricular "Ecografía en Cirugía General", aplicándose un diagnóstico inicial, luego se impartió el Taller Docente y finalmente se aplicó una prueba final, validándose así la utilidad de dicha estrategia curricular. Resultados: Se obtuvo mejores calificaciones en la prueba final con relación al diagnóstico inicial, dadas por, elevación del promedio de notas de 2,8 a 4,3 puntos; superioridad en el índice de calidad, manifestándose por el aumento de estudiantes con calificaciones entre 4 y 5 puntos de 8 a 100 y disminuyéndose el total de suspensos de un 30,8 a un $0 \%$ respectivamente. Conclusiones: La Estrategia curricular: Taller Docente "Ecografía en Cirugía General" como medio de enseñanza constituyen un valioso aporte para la adquisición de conocimientos dentro del proceso docente-educativo, por las grandes posibilidades que brinda para facilitar el aprendizaje.

Experiencia Inicial En El Uso De Cirugía Mínimamente Invasiva En El Diagnóstico Y Tratamiento Del Paciente Con Trauma Penetrante Abdominal. Juan PR Perkis, Pablo RO Lavarte, José ER Cabrera, Analía ZAcosta, Claudia, Carolina M Alarcon, Pablo A Tirado. From the Unidad De Trauma Y Urgencias Hospital Dr Sótero Del Río., Santiago, Chile. 
Introducción: En las últimas décadas la cirugía mínimamente invasiva (CMI) como método diagnóstico y terapéutico ha aumentado las herramientas de manejo del paciente con trauma. El objetivo de este trabajo es presentar los resultados obtenidos en pacientes con trauma penetrante abdominal sometidos a laparoscopía (LPC) posterior a implementar un protocolo de CMI en la unidad de trauma y urgencias del Hospital Dr Sótero del Río. Materiales y métodos: Estudio descriptivo retrospectivo de diciembre del 2017 a marzo 2019 que incluye pacientes hemodinámicamente estables y con criterios de inclusión para protocolo de CMI. Se utiliza el registro de trauma del servicio de salud metropolitano sur oriente para análisis de variables. Resultados: Serie compuesta por 61 pacientes víctima de trauma penetrante sometidos a CMI. El $94 \%$ son hombres. El $70,7 \%$ ingresa por trauma por arma blanca y $29,2 \%$ por arma de fuego. El $90 \%$ tiene un hallazgo patológico en la tomografía. Se logra una cirugía totalmente laparoscopica en el 90,2\% teniendo un $9,7 \%$ de conversión. En el $51,2 \%$ de los pacientes la laparoscopía es terapéutica, realizando 12 procedimientos hemostáticos, 7 frenorrafias, 7 suturas intestinales, 3 gastrorrafias, 2 ostomias, 1 anastomosis y cistorrafia. En el 39,2\% se realiza laparoscopía diagnostica. Los órganos mas afectados son diafragma (7), Hígado (9), Intestino delgado (5) y colon (3). Las seis causas de conversión son por dificultad técnica del cirujano. La media de hospitalización es de 3,8 días (rango 2-8 días). El 6\% del total presenta una complicación dentro de su hospitalización. No existe en la serie lesiones desapercibidas ni mortalidad. Conclusiones: La CMI es una opción real en el diagnóstico y tratamiento de pacientes víctima de trauma penetrante hemodinámicamente estables. La adecuada selección de pacientes es el factor principal para la aplicación con seguridad de ésta técnica y así tener una corta estadía hospitalaria y baja morbilidad.

Massive Transfusion. Is It Time for a New Definition? Daniela Burbano, Julian Chica, Claudia Orlas, Ramiro Manzano, Sandra, Camilo Salazar, Fredy Ariza, Alberto García. From the Fundación Valle del Lili-Universidad Icesi, Valle Del Cauca/Cali, Colombia.

Introduction: Massive transfusion (MT) is defined as the administration of -10 units (U) of packed red blood cells (PRBC) in 24 hours. Alternative definitions have been proposed which haven't been compared regarding mortality or multiorgan failure (MOF). The objective is to compare the discriminative ability of proposed definitions of MT concerning mortality and MOF. Materials and methos: Patients with trauma team activation in a Level I trauma hospital of Cali, Colombia between 2014 and 2018 were included. Demographics and trauma characteristics were evaluated. The following MT definitions were measured: $50 \mathrm{U}$ of blood products in 24 hours (T50), $10 \mathrm{U}$ PRBC in 24 hours (T10-24), $6 \mathrm{U}$ PRBC in 6 hours (T6-6), $10 \mathrm{U}$ PRBC in 6 hours (T10-6), combination of T10-24 and T6-6 (T-combi), 5 U PRBC in 4 hours (T5-4), $4 \mathrm{U}$ PRBC in 1 hour (T4-1) and $3 \mathrm{U}$ PRBC in 60 minutes. The operative characteristics were calculated for each definition. MOF was defined as a SOFA score of 6 points. Results: We included 394 subjects, $88.6 \%$ male. Trauma mechanism was penetrating in $87.3 \%$. The median and interquartile range (IQR) of age was 28 years (22-37) and of ISS 25 (16-26). Lesions were located in the torso in $42.4 \%$ individuals, and $47.2 \%$ had a positive ABC score. A total of 264 (67\%) received at least 1 unit of PRBC. Sensitivity, specificity were as follows: MOF: T10-24 16.67\% and $99.11 \%$, T6-6 30.70\% and $95.56 \%$, T-combi $34.21 \%$ and $95.56 \%$, T5-4 34.21\% and 96\%, T4-1 42.98\% and 88.89\%. Mortality: T10-24 $35.80 \%$ and $95.11 \%$, T6-6 $55.22 \%$ and $89 \%$, T-combi $56.72 \%$ and $87.77 \%$, T5-4 $53.73 \%$ and $88 \%$, T4-1 $62.70 \%$ and $80.12 \%$. Conclusion: All definitions showed an association with a higher risk of mortality and MOF, generally with low sensitivity but high specificity. The MT definition of-10 packed red blood cells (PRBC) in 24 hours should be revised.

\section{Manejo Mínimamente Invasivo De Paciente Estable Con Trauma Precordial Penetrante. Juliana M Ordoñez, Daniel Gonzalez, Diego FC Medina, Mauricio Pedraza, Luis Felipe, Tabata L Tinoco, Orlando Arevalo, Adolfo Gonzalez, David Giraldo, Gregorio Gonzalez. From the Hospital Universitario Del Valle Evaristo Garcia, Cali, Colombia.}

Introducción: El trauma cardiaco en países subdesarrollados es de predominio penetrante, con altas tasas de mortalidad extrahospitalaria pero que en el ámbito hospitalario dependerá en parte de si el paciente se encuentra taponado o no. El enfoque inicial de los pacientes con trauma precordial penetrante se simplificó considerablemente, en la medida en que permitió evitar toracotomías innecesarias. Las técnicas mínimamente invasivas para practicar la ventana pericárdica y la nueva interpretación del resultado positivo han hecho variar paradigmas previamente establecido, lo cual ha llevado a realizar un cambio en el abordaje inicial de los pacientes con trauma precordial penetrante hemodinamicamente estables. Materiales y métodos: Objetivo: Mostrar nuestra experiencia en cirugía mínimamente invasiva de pacientes hemodinamicamente estables llevados a ventana pericárdica por trauma precordial. Materiales: Pacientes ingresados con herida penetrante precordial, estables, al servicio de trauma Hospital Universitario del Valle Evaristo García; revisión historia clínica y literatura. Resultados: Pacientes con E-FAST+ subxifoidea; llevados a ventana pericárdica por toracoscopia y laparoscopia, todas positivas con identificación de lesiones en pericardio, aurícula derecha, ventrículo izquierdo y aurícula izquierda no sangrante. Manejo con lavado con SSN 500 cc hasta obtener líquido claro, aspiración de coágulos. Drenaje de hemotórax y toracostomia cerrada en abordaje torácico. Retiro de tubo de tórax 72 horas después y egresó sin complicaciones documentadas. Conclusiones: La cirugía mínimamente invasiva en trauma resulta ser efectiva y segura para el manejo del trauma penetrante precordial en el paciente estable hemodinamicamente, evitando toracotomías y estereotomías innecesarias. Es necesario que el personal esté entrenado en cirugía mínimamente invasiva y se escoja al paciente indicado.

Using Critical Administration Thresholds (Cat) to Predict Damage Control Surgery in Patients with Non-Compressible Torso Injuries. Natalia Padilla, Claudia P Orlas, Cheryl K Zogg, Maria A Londoño, Juan, Camilo J Salazar, Diana Martinez, Alberto F Garcia, Michael W Parra, Carlos A Ordoñez. From the Fundación Valle del Lili, Cali, Colombia.

Introduction: Critical Administration Thresholds (CAT) is a novel method used to define large-volume transfusion, accounting for rate 
and volume simultaneously. CAT may also serve as a simple trigger to distinguish patients benefiting from damage control surgery (DCS). The purpose of this study was to determine if CAT was predictive of the need for DCS in patients with non-compressible torso injuries (NCTI). Materials and methods: All trauma patients with NCTI from 2014 to 2017 at a level I trauma Center were included. Patients were classified by the number of times that they met CAT ( $3 \mathrm{U}$ of blood/1 hour during day 1 ) over the span of 24 hours. Basic demographics, clinical variables, time to CAT+ status and DCS requirement were analyzed. Results: A total of 400 patients were included. 234 underwent DCS, and 349 (87.2\%) had penetrating trauma. DCS patients were more unstable upon arrival to the Emergency Room [Non-DCS = SBP: 106 mm Hg (IQR: 80-123), HR: 96 bpm (IQR: 77-110), vs DCS = SBP: 89 mm Hg (IQR: 60-110), HR: 110 bpm (IQR: 88-127); p. Conclusion: In NCTI patients, the CAT+ status (CAT-1/CAT-2), ISS value and lactate levels predicted DCS requirement. As such, CAT+ status serves as a logical early trigger to identify patients benefiting from DCS.

Shock Index in Patients with Traumatic Pelvic Fracture: Insights from a Level 1 Trauma Center. Ayman El-Menyar, Husham Abdelrahman, Hassan Al-Thani, Sandro Rizoli. From the Hamad general hospital, Doha, Qatar.

Introduction: Pelvic injuries and other associated injuries pose various diagnostic and therapeutic challenges for the front-line emergency physicians. We aimed to assess the utility of shock index (SI) to predict the need for blood transfusion and outcome in patients with traumatic pelvic fracture (TPF) at a level I trauma center. Materials and methods: We conducted a retrospective analysis for all patients with TPF between 2012 and 2016. Patients were categorized into low vs high SI based on the cut-off on the receiver operating characteristic (ROC) curves that were plotted to predict mortality. Results: A total of 966 patients sustained TPF (28.5\% had had SI -0.9 based on ROC) with a mean age of $36.7 \pm 15.6$ years. Type $B$ and $C$ fractures significantly had high SI. The frequency of blood transfusion, and amount of blood ( -10 units) needed were more in patients with high SI $(p=0.001)$. SI was correlated significantly with ISS $(r=0.32)$, RTS $(r=-0.40)$ and transfused blood units $(r=0.35)$. In-hospital pneumonia, sepsis and acute kidney injury were more in the high SI group $(p=0.001)$. Patients with high SI had prolonged hospital stay and higher mortality than those with low $\mathrm{SI},(p=0.001)$. SI -0.9 had high sensitivity and negative predictive value to identify the need for massive blood transfusion-10 units ( $77 \%$ and $86 \%$, respectively) and mortality ( $73.5 \%$ and $98.1 \%$ respectively). For mortality, high SI had sensitivity $73.5 \%$, specificity $74 \%$, negative predictive value $98 \%$, and negative likelihood ratio of 0.36 . After adjustment for age, sex, ISS,GCS, pelvis AIS, blood transfusion and Tile classification, multivariate analysis models showed that high SI was independent predictor of blood transfusion (OR 5.6) and mortality (OR 3.63). Conclusion: This is a large series study that assesses the clinical implications of SI in patients with pelvic fractures. SI performed well for prediction of massive transfusion and mortality in patients with TPF.

¿Ha Ccambiado La Epidemiología De La Diverticulitis Aguda En Los Servicios De Urgencia? Experiencia Del Hospital Urgencia Asistencia Pública, Santiago De Chile. Heinz Dauelsberg, Vicente Hernández, Mónica Martinez. From the Hospital de Urgencia Asistencia Pública, Santiago, Chile.
Introducción: La diverticulitis es un diagnóstico frecuente en urgencia. Algunos estudios sugieren un aumento en la incidencia, especialmente en países industrializados y en menores de 50 años. El objetivo es analizar las variables sociodemográficas, clínicas y desenlace de pacientes con diverticulitis aguda ingresados a nuestro Hospital, con énfasis en diverticulitis complicada. Materiales y métodos: Estudio descriptivo de cohorte de 368 pacientes ingresados con diagnóstico de diverticulitis aguda ingresados al HUAP entre los años 2011-2016. Se estudiaron las variables sociodemográficas y clínicas de estos pacientes. Resultados: Entre 2011 y 2016, de 54.454 egresos totales, 368 $(0.7 \%)$ corresponden a diverticulitis aguda, predominando el sexo femenino con 240 pacientes (65\%). La edad media fue 61 años. No hubo diferencias significativas respecto a la frecuencia ni edad de los pacientes al separarlos por año. La estadía fue 6.9 días. La tasa de reingreso general fue $2.7 \%$, con mortalidad general del $3.5 \%$. Del total, 88 (24\%) corresponden a diverticulitis complicada, $58 \%$ son mujeres y $42 \%$ hombres. De estas $26,1 \%$ son menores de 50 años, siendo hombres el $78 \%$. De las complicaciones, el $51,13 \%$ corresponden a perforación, siendo $12,2 \%$ del total. El $36,4 \%$ presentó absceso, siendo $62,5 \%$ sigmoideos. El $11,36 \%$ presento fístulas, de estas, $40 \%$ corresponden a fístula colovesical y $1,1 \%$ obstrucción intestinal. Se clasificó como Hinchey $(31,8 \%)$, Hinchey II $(20,4 \%)$, Hinchey III $(30,6 \%)$ y Hinchey IV $(15,9 \%)$. De las diverticulitis complicadas, se realizó manejo médico en el $39,8 \%$ y quirúrgico en $60,2 \%$. Correspondiendo a cirugía tipo Hartmann 23,86\%. El 15,9\% requirió reintervención quirúrgica. De las diverticulitis complicadas, el 76,1\% presentó SIRS, $30,6 \%$ sepsis y un $17,1 \%$ presentó falla orgánica múltiple. La tasa de mortalidad de diverticulitis complicada fue $9 \%$. Conclusiones: Destaca el aumento de la diverticulitis perforada y el aumento de incidencia en menores de 50 años (26,1\%), siendo principalmente hombres. La mayoría de las Diverticulitis perforadas tuvo manejo quirúrgico.

Independência Para Realizar as Atividades de Vida Diária Após - Trauma Cranioencefálico Grave. Rita DCAV Vieira, Wellingson DSP Paiva, Daniel VDO Oliveira, Regina MCDS Sousa. From the Universidade de São Paulo, São Paulo, Brazil.

Introducción: Estudar a independência para realizar as atividades de vida diária (AVD) é importante para ampliar o conhecimento nessa área e monitorar a evolução das vítimas. Objetivo da pesquisa foi verificar a independência para realizar AVD e atividades instrumentais de vida diária (AIVD) em vítimas de TCE grave doze meses após o trauma. Materiales y métodos: Estudo do tipo follow-up, com dados coletados na internação, alta, três, seis e doze meses após o TCE grave. Fizeram parte do estudo vítimas de TCE grave (Escala de Coma de Glasgow -8) com idade -18 anos e -60 anos, admitidas no Hospital das Clínicas da Faculdade de Medicina da Universidade de São Paulo. A independência nas AVD foi analisada pelas diferenças dos resultados da aplicação da escala de Katz na alta, 3 , 6 e 12 meses e da escala de Lawton aos 3, 6 e 12 meses após TCE. Para comparar a média da pontuação total da escala de Katz foi utilizado a análise de variância (ANOVA) e para AIVD foi aplicado o teste não paramétrico de Friedman com medidas repetidas. Resultados: A casuística compôs-se de 228 vítimas, com idade média de 32,4 anos ( $d p=12,1), 57,9 \%$ envolvidas em 
acidente de transporte, $89 \%$ do sexo masculino. Até 12 meses, 71 pacientes foram à óbito, a pontuação média do Injury Severity Score dos sobreviventes foi de 31,5 (dp $=8,7)$ e a pontuação média da Katz dos sobreviventes foi de 8,6 (dp = $6,6)$ na alta para $1,6(\mathrm{dp}=4,5)$ na avaliação final. Para escala de Lawton a média foi de $17(\mathrm{dp}=7,4)$ para 19,4 $(\mathrm{dp}=7,6)$ na avaliação final. Diferenças estatisticamente significativas foram observadas na comparação dos resultados de todos os tempos. Conclusiones: Foi elevada a mortalidade até doze meses após o TCE grave, entanto, os sobreviventes alcançaram escore médio compatível com independência para realizar AVD e AIVD aos doze meses após lesão.

Laparotomía De Control De Daños En Peritonitis Secundaria a Enfermedad De Viscera Hueca: Es Seguro Diferir La Reconstrucción Intestinal? Juan JMelendez, Claudia POrlas, Kimberly Alcazar, Juan J Martinez, Juliana, Carlos A Serna, Alexander Salcedo, Alberto F García, Michael W Parra, Carlos A Ordoñez. From the Fundación Valle del Lili, Cali, Colombia.

Introducción: La cirugía de control de daños se ha integrado en el armamento de la cirugía de emergencias, su aplicación en peritonitis y abdomen catastrófico todavía no es bien conocida. El objetivo de este estudio fue evaluar la factibilidad y aplicabilidad de la reconstrucción intestinal diferida en pacientes con peritonitis secundaria a compromiso de viscera hueca. Materiales y métodos: Todos los pacientes de No-trauma (edad $>18$ años) que ingresaron con sospecha de peritonitis y que se sometieron a laparotomía entre enero/2004 y diciembre/2018 fueron incluidos. Las características sociodemográficas, comorbilidades, escalas de severidad fisiológica, técnicas de reconstrucción intestinal y, desenlaces clínicos fueron evaluadas. Resultados: 716 pacientes fueron identificados, 410 no fueron elegibles. De 306 pacientes, 120 (39.2\%) se sometieron a Resección y Anastomosis [grupo I], 87 (28.4\%) a Ostomía [grupo II] y en 99 (32.3\%) se realizó Ligadura Intestinal [grupo III]. Los pacientes sometidos a Ligadura presentaron mayor Apache al ingreso a UCI [Apache II, grupo I = 14 (IQR: 10-18), grupo II = 13 (IQR: 11-18), grupo III = 18 (IQR: 14-24), p. Conclusiones: La laparotomía de control de daños con ligadura intestinal logró evitar la realización de ostomía como técnica de reconstrucción definitiva en más de la mitad de los pacientes. Implementar esta técnica dentro del campo de la cirugía de cuidado agudo es factible y seguro en pacientes con compromiso fisiológico y anatómico severo.

Análise Epidemiológica Das Vítimas De Trauma Cardíaco Em Um Hospital De Referência. Christina NSC Nahrari, Iwan ACI Collaço, Lucas MSL Sarquis, Lucas FL Favero, Fábio De Carvalho, Rodrigo KMR Krieger, Caroline LBDBCDal Bosco, Matheus SEMS Evangelista, Arnon CBSACB Schultze, Bruno BGBB Gazola. From the Hospital do Trabalhador, Curitiba-Pr, Brazil.

Introducción: Analisar o perfil epidemiológico dos pacientes vítimas de trauma cardíaco submetidos à cardiorrafia. Este é um estudo retrospectivo e transversal de casos de pacientes com lesões cardíacas traumáticas, entre janeiro de 2017 e janeiro de 2019, que foram submetidos à cardiorrafia, em um centro de referência de trauma em Curitiba/PR-Brasil.
Materiales y métodos: Todos os casos foram decorrentes de vítima urbana, sendo nove com ferimentos penetrantes (seis $F A B$, três FAF) e um trauma contuso com agressão física. $80 \%$ eram homens, com idade média de 37,6 anos. Resultados: Nove casos ocorreram entre sexta-feira e segundafeira, sendo $70 \%$ no período noturno. Cinco pacientes foram admitidos hemodinamicamente estáveis e cinco instáveis. Do total, $40 \%$ foram diagnosticados com FAST, o restante necessitou de tomografia computadorizada e outros exames de imagem. As incisões utilizadas foram esternotomia mediana em 50\% pacientes e toracotomia ântero-lateral esquerda nos outros $50 \%$. Das lesões encontradas, cinco foram em Ventrículo Direito (VD), duas em Átrio Direito (AD), duas em Ventrículo Esquerdo (VE) e uma em parede anterior e ápice. Todos foram submetidos à cardiorrafia. Oito pacientes receberam alta e os dois que se apresentaram hemodinamicamente instáveis à admissão evoluíram a óbito. O ecocardiograma dos oito pacientes, no pós-operatório, apresentou valores de fração de ejeção entre 55,1\% e 75\% (média $65,78 \%$ ), com tempo médio de UTI de 8,1 dias e de internamento total 12,1 dias, encaminhados para seguimento ambulatorial. Conclusiones: $O$ estudo tornou evidente a predominância de ferimentos cardíacos entre homens, por motivo violento, durante os finais de semana. A taxa de mortalidade está em consonância com a encontrada na literatura (20\%) e o tratamento por cardiorrafia mostrou-se eficaz, especialmente nos casos de admissão com estabilidade hemodinâmica, independentemente do tipo de incisão escolhida para reparo.

Perfil Epidemiológico De Pacientes Submetidos a Nefrectomia Após Trauma Renal Em Hospital Referência Em Trauma De CuritibaPr. Thamyle MDS Rezende, Iwan A Colaço, Fábio De Carvalho, Hector Fontes, Lucas, Henrique DA Wiederkehr, Caroline LBD Bosco, João OV Zeni, Lucas Favero, Kauana O Gouveia. From the Hospital do Trabalhador, Curitiba-Pr, Brazil.

Introducción: Analisar epidemiologicamente os casos de lesão renal traumática grave submetidos a tratamento cirúrgico em um hospital referência em trauma de Curitiba-PR. A partir de coleta de dados do prontuário eletrônico foram identificados no período de janeiro/2017 a janeiro/2019 os pacientes submetidos a nefrectomia em hospital referência em trauma de Curitiba-PR. Materiales y métodos: Foram analisados 17 pacientes, sendo $94,1 \%$ masculinos e com média de idade de 25,23 anos (16 a 44). 0 trauma contuso ocorreu em 9 casos $(52,9 \%)$ e o penetrante em $8(47,1 \%)$. Dentre os contusos, 77,77\% foram devidos a acidente automobilístico. Resultados: As lesões foram estratificadas de acordo com o American Association for the Surgery of Trauma (AAST) estando entre os graus III $(23,5 \%)$, IV $(35,3 \%)$ e grau V $(41,2 \%)$. Tomografia de abdômen foi realizada em 12 casos (70,5\%), fornecendo o diagnóstico de lesão renal em 9 desses contra 8 diagnósticos intraoperatórios. Em 14 pacientes realizou-se transfusão sanguínea com média de $3107,6 \mathrm{~mL}(250$ a 8,650). As principais lesões associadas ocorreram em fígado (52,9\%), baço $(35,2 \%)$ e crânio-encéfalo $(5,8 \%)$. Foi tentado tratamento conservador em 3 casos, todos evoluíram para nefrectomia. Cirurgia de controle de danos foi realizada em 10 pacientes. A média de reabordagens foi 1,05 $(0 ; 4)$. Foram internados em UTI 82,35\%, com tempo médio de 20,23 dias (4 a 95). Apenas 3 pacientes evoluíram com complicações pós-operatórias com pequenos sangramentos e 2 foram a 
óbito. Em nenhum dos casos houve alteração da função renal durante seguimento ambulatorial com necessidade de diálise. Conclusiones: Pacientes masculinos sofrem mais traumas resultantes em lesão renal, sendo aquelas com necessidade de nefrectomia as de grau III ou superior. Os pacientes apresentaram altas taxas de internação em UTI, porém baixas taxas de complicações e morbi-mortalidade.

Scores De Trauma vs Score De Enfermedad Crítica En Pacientes Traumatizados Críticamente Enfermos. Análisis De Un Registro De Trauma De Un Hospital Pediátrico. Andrea Francavilla, Adriana Simons, Deborah Turina, Alejandro Gattari, Virginia, Ignacio Bari, Ezequiel Monteverde, Pablo Neira. From the Hospital de Niños Ricardo Gutiérrez, Buenos Aires, Argentina.

Introducción: El trauma es la principal causa de muerte en niños. Aproximadamente el $10 \%$ de los ingresos a unidades de cuidados intensivos (UCIPs) se relacionan con esta causa. Establecer una forma de evaluación común de los resultados en UCIPs es un factor crítico para la mejora de la calidad. En esta población no existe certeza si los scores de enfermedad crítica o los específicos de trauma son los mejores predictores de desempeño. Materiales y métodos: Revisión retrospectiva del registro de trauma de la UCIP del Hospital de Niños Ricardo Gutiérrez, que incluye datos deidentificados de todos los ingresos a la unidad entre 2005 y 2017. Los scores evaluados fueron: índice de trauma pediátrico (ITP), score de severidad lesional (ISS), nuevo score de severidad lesional (NISS), score de trauma revisado (RTS), score de severidad de trauma y lesiones (TRISS) e índice de mortalidad pediátrica (PIM2). Resultados: Se incluyeron 360 pacientes (56\% varones) con una edad de 60 meses, 30-116 (mediana, RIC). 73\% tuvo lesiones intracraneanas, $26 \%$ tuvo compromiso de extremidades, $19 \%$ tórax, $14 \%$ abdomen, $6 \%$ pelvis y $5 \%$ columna vertebral. $43 \%$ tuvo lesiones en una región corporal, $29 \%$ en dos y $28 \%$ en más de dos. La combinación más frecuente fue cabeza y extremidades (16\%). Las principales causas fueron caídas (42\%), peatones $(20 \%)$ y ocupantes de automóviles (7\%). La mortalidad general fue $5.3 \%$. Para predecir el riesgo de muerte en UCIP se probó la capacidad de discriminación de los scores usando curva ROC (ABC e IC95\%): NISS 0.749 (0.63-0.86), ISS 0.79 (0.69-0.89), PTS 0.90 (0.84-0.96), RTS 0.912 (0.84-0.98), TRISS 0.930 (0.86-0.99) y PIM2 0.977 (0.93-1.0). Conclusiones: En esta muestra de una única institución, el score PIM2 tuvo una capacidad de discriminación superior a los scores de trauma usando mortalidad como variable resultado. Estos resultados necesitan probarse en una población más grande.

Perfil Dos Paciente Vítimas De Trauma Hepático Com Tratamento Cirúrgico Em Hospital De Referencia. Barbara V Sardi, Iwan A Collaço, Eduardo L Martins, Adonis Nars, Lucas, João GS Lemes, Henrique DA Wiederkehr, João OV Zeni, Bruna Pignataro, Carmen $M$ Jamur. From the Hospital do Trabalhador, Curitiba-Pr, Brazil.

Introducción: O objetivo deste trabalho é descrever o perfil dos pacientes com lesão hepática pós trauma, identificando o procedimento realizado bem como a incidência de complicações cirúrgicas associadas. Foi realizado um estudo transversal avaliando os pacientes com lesão hepática pós trauma no período de janeiro de 2017 a janeiro de 2019. Materiales y métodos: Foram incluídos 38 pacientes e coletadas variáveis epidemiológicas e relacionadas ao trauma, sendo excluídos pacientes submetidos a tratamento conservador ou aqueles sem dados suficientes no prontuários eletrônicos para análise proposta. Resultados: Dos 38 pacientes, 35 homens e 3 mulheres, 92,1\% estavam na faixa etária dos 15-65 anos. O mecanismo predominante foi ferimento por arma de fogo (68,4\%). O diagnóstico das lesões em $44,7 \%$ foram feitas a partir da Tomografia Computadorizada, sendo as principais hepática grau III/IV e associadas as de diafragma e gástrica. Foi optado por cirurgia no manejo inicial devido a instabilidade hemodinâmica ou lesões que necessitavam de abordagem cirúrgica. Esses pacientes foram submetidos a Hepatorrafia, Segmentectomia hepática, Hepatectomia não regrada ou eletrocoagulação com procedimentos associados ou não entre si. O principal procedimento realizado foi a Hepatorrafia (62,2\%). Dentre 38 pacientes, 2 faleceram no intraoperatório, 31 foram encaminhados para a Unidade de Terapia Intensiva, sendo a média do tempo de internamento de 20,6 dias, 5 foram encaminhados para enfermaria, com média de 7,8 dias. Entre os casos descritos, 11 pacientes foram submetidos a cirurgia de controle de danos para tratamento definitivo em um segundo momento. Sobre complicações cirúrgicas, as principais foram deiscência (13\%) e fístula biliar (13\%). Do total, 8 foram a óbito e 30 tiveram altas com seguimento ambulatorial. Conclusiones: As lesões hepáticas que necessitem de tratamento cirúrgico estão associadas com necessidade de cirurgia de controle de danos. A fistula biliár e o óbito são complicações possíveis, seja pela própria lesão hepática ou pelas lesões associadas.

Management of Mild Traumatic Brain Injury in an Acs-level III Trauma Center. The Wilmington, De Experience. Jaime CG Arango, Mary Baratelle, Mark Cipolle, Pam Woods, Marilynn, Joan Pirrung. From the Christiana Care Health System, Delaware, USA.

Introduction: Wilmington hospital $(\mathrm{WH})$ became an ACS-verified Level III trauma center in October 2016, motivated to reduce the burden of managing low to moderately injured patients transferred to the system's ACS-verified Level I trauma center, Christiana hospital $(\mathrm{CH})$. We hypothesized that retaining mild TBI patients at WH would be an integral part of this new Level III trauma center. Materials and methods: Data was collected from the WH trauma registry from Oct/2016-March/2019. Evaluated variables were: number of evaluations, number of admissions, ISS, head trauma, mortality and transfers. Chi square analysis was used. Results: Opening the doors of WHTC October/2016 resulted in a reduction in transfers and admissions to $\mathrm{CH}$ in 2017. In 2017, WH entered 855 patients into their trauma registry resulting in 513 admissions, 501 (97.7\%) with ISS 1-15. In 2018 our admissions were 1,025 and more importantly, WH demonstrated that it was likely effective in off loading the low to moderate injury patients from $\mathrm{CH}$, some of the most significant have been the mild TBI patients. All head injuries from 10/1/2016 to 03/31/2019 were included. The total number of patients evaluated by the trauma service since its inception in October/2016 until March/2019 was 2300, of those 264 (11\%) were transferred. A total of 375 (16.3\%) were patients with traumatic brain injury, 90 of those (24\%) were transferred, excluding concussion 278 patients were evaluated and 79 (28\%) transferred. Mortality was 0. Conclusion: Mild TBI could be managed at basic level trauma centers with a careful coordination of care for neurosurgery referrals as indicated to a Level ITC. The development of the WH Level III trauma 
center, in a nearby urban area, resulted in refinement of criteria for transfers for TBI patients. We are improving the premise of "right patient, right place, right time" in the DETrauma System and following TQIP recommendations.

Catecholamine Surge after Traumatic Brain Injury: A Window of Opportunity. Airton L De Oliveira Manoel, Sandro Rizoli, Ruben Peralta. From the Hamad General Hospital, Doha, Qatar.

Introduction: Although no current pharmacological treatment is available to reduce the progression of secondary brain injury after traumatic brain injury (TBI), the use of beta-blockers has shown promising results. We evaluated the literature on the association between catecholamine levels and outcome, as well as the potential role of beta blockage after TBI. Materials and methods: We performed a systematic review using MEDLINE, EMBASE, and Google Scholar. Eligible articles that described the association between catecholamine levels and outcome, as articles that tested the use of beta-blockers after TBI were searched. Results: Five cohorts described the association between catecholamine levels, the severity of TBI and functional outcome. High catecholamine levels were associated with severity of brain injury, longer duration of mechanical ventilation and length of hospital stay. The larger cohort found included 174 patients, which showed that high catecholamine levels were independently associated with unfavorable functional outcome. High epinephrine levels were associated with a higher risk of unfavorable outcome (OR, 2.04, 95\% Cl: 1.31-3.18, $p=0.002)$ and mortality (OR, 2.86, 95\% Cl: 1.62-5.01, $p=0.001)$. Regarding the use of beta blockage, only one randomized trial was found. This study tested the use of atenolol on cardiac morbidity after TBI. Atenolol was associated with lower likelihood of cardiac events, such supraventricular tachycardia or ST-segment changes. Fourteen cohorts that reported the association between the use of ?-blocker and mortality were found. Twelve of them showed lower adjusted odds of mortality. One matched case-control study described the association of ?-blocker and functional outcome. The risk of unfavorable functional outcome was more than doubled without the use of beta-blocker (OR 2.44, 95\% Cl 1.01-6.03, $p=0.03$ ). Conclusion: High catecholamine levels after TBI is associated with worse outcome. The use of beta-blocker may reduce mortality and improve long-term outcome. These benefits must be adequately evaluated in a multicenter clinical trial.

Epidemiologia De Vítimas De Projétil De Arma De Fogo Da Sala De Trauma Em Hospital De Urgência Na Zona Oeste Do Rio De Janeiro Entre 2015 E 2017. Rafael Zigliotti, Ana B Rocha, Samy Chitayat. From the Universidade Unigranrio, Rio De Janeiro, Brazil.

Introducción: Nas últimas décadas, no Brasil, observou-se um aumento da violência. Estudos revelam que no período de 1980 e 2010, aproximadamente 800 mil pessoas morreram vítimas de arma de fogo, dentre elas o principal grupo acometido foram os jovens entre 15 e 29 anos. Nesse período houve um crescimento de $346,5 \%$. O objetivo do trabalho é avaliar o perfil epidemiológico das vítimas de ferimento por arma de fogo em hospital de urgência. Materiales y métodos: Trata-se de um estudo ecológico, observacional, transversal, retrospectivo, com abordagem na investigação territorial, mediante coleta de dados de 26.677 pacientes, realizada por consulta ao sistema de cadastro de pacientes que são admitidos na sala de trauma do Hospital Municipal Lourenço Jorge localizado na Zona Oeste do Rio de Janeiro, Brasil. Período da coleta dos dados: janeiro de 2015 a dezembro de 2017. Resultados: Os pacientes vítimas de lesão por arma de fogo representaram 143 casos em 2015, 231 em 2016 e 251 em 2017, totalizando 625 casos neste período. Os mais acometidos foram do sexo masculino, representando 91\%, na faixa etária de 20 a 29 anos. Houve maiorocorrência do trauma no período de 00 horas às 04 horas da manhã. Conclusiones: Em correlação com outros estudos, a incidência mostrou-se marcante em regiões que abrangem a presença do tráfico de drogas, refletindo o contexto da violência interpessoal na sociedade atual, mantendo relação com o território em que o Hospital Municipal Lourenço Jorge abrange, rodeado de inúmeras comunidades carentes dominadas pelo tráfico de drogas. Diante da magnitude do trauma ocasionado por projétil de arma de fogo, ressalta-se a importância de uma equipe de cirurgiões de trauma treinados em lesões por PAF de diferentes complexidades e não somente cirurgiões gerais, nos hospitais de Urgência.

Abordagem Do Choque Hemorrágico No Paciente Politraumatizado: Treinamento Em Simulador De Baixo Custo. Paulo GS Da Silva, Santiago CN Servin, Artur AS Batista, Larissa $R$ De Oliveira Sales, Ingrid, Lais MG Coelho, Bárbara NC Dos Santos, Andrey SM Filho, Rodrigo MS Alves, Micael MV Silva. From the Universidade Federal do Maranhão, Maranhão/São Luís, Brazil.

Introducción: A simulação realística na faculdade dá aos estudantes a oportunidade de desenvolverem habilidades variadas e competências necessárias em ambiente controlado e protegido. Permite erros e crescimento profissional, sem arriscar a segurança do paciente. Esse trabalho tem como objetivo descrever treinamento em situações dechoque hemorrágico através de simulador de baixocusto. Materiales y métodos: $O$ treinamento foi desenvolvido durante um curso sobre trauma oferecido pela Liga de Trauma a acadêmicos de medicina. Dentre as estações práticas do curso, tem-se a estação do choque. A prática de punção venosa é realizada com jelco em simulador desenvolvido a partir de sonda de Foley, luva de borracha e um sistema preenchido com corante vermelho; a sonda é inserida na luva e fixada de modo a se apresentar semelhante a um vaso sanguíneo e tem sua extremidade acoplada no sistema; realiza-se então a punção com o Jelco e, ao aspirar, tem-se a certificação do sucesso ou insucesso da técnica. Para simular a punção intraóssea, utiliza-se pés de galinha, agulhas de tamanho $18 \mathrm{G}$ e corante azul; punciona-se a superfície anterior do membro inferior da galinha, representando uma sensação tátil semelhante a transfixação da tíbia; após isso injeta-se a solução azul e então, com o extravasamento dessa pela medula óssea, confirma-se o sucesso da punção. Resultados: $O$ modelo foi bem aceito, tendo uma média 8 ao estabelecer a correlação anatômica entre o simulador e a anatomia humana. Foi observado um acréscimo de conhecimento ao longo da estação prática. A estação vem sendo bem avaliada pelos participantes, pela sua metodologia de ensino e prática, uma vez que se aproxima bastante de situações realísticas. Conclusiones: Percebe-se que a associação do método tradicional com a simulação realística mostra-se eficaz para a consolidação do conhecimento. O modelo foi capaz de representar uma situação de punção venosa e óssea próxima à realidade, sendo eficaz para treinamentos. 
Prior Emergency Department Utilization as a Predictor for Severe Penetrating Trauma: A Retrospective Cohort Study. Alexandra Reitz, Stephen Pitts, Michael Goodman. From the Emory University School of Medicine, Atlanta, Ga, USA.

Introduction: Penetrating trauma is a leading cause of mortality among adults under the age of 65 . While extensive research has been conducted on healthcare utilization subsequent to severe penetrating trauma events, there is a dearth of information on healthcare utilization prior to these events. This study examined emergency department (ED) utilization patterns to determine if prior ED visits for injury were a risk factor for severe penetrating trauma. Materials and methods: This retrospective cohort study examined the ED visit records of 215,800 patients with 489,800 ED visits and 3,322 trauma registry patients from November 2010 to February 2015 at a large, urban hospital with a Level I trauma center. Data analysis was conducted using logistic regression and Cox proportional hazard models. Results: Among 215,800 ED patients, 224 patients with prior ED visits experienced severe penetrating trauma. In a bivariate model, prior ED visits for injury (OR 2.17, Cl 1.67-2.83, $p<0.0001$ ) were significantly associated with severe penetrating trauma. After adjustment for age, gender, employment, insurance, high utilization, and admission status, prior ED visits for injury continued to be associated with severe penetrating trauma (OR $1.60, \mathrm{Cl}$ 1.21-2.10, $p=0.001)$. Cox proportional hazard models estimated hazard ratios (HR) for factors associated with time to a penetrating trauma event following a patient's last ED visit. After adjusting for age, gender, employment status, admission status, high utilization, and insurance status, patients with an injury diagnosis at their last ED visit had a HR of 1.43 (Cl 1.07-1.93, $p=0.016$ ). Conclusion: After adjusting for confounders, a previous ED visit for injury remained a significant risk factor for severe penetrating trauma. Patients with a last ED visit for injury also had an elevated rate of time to penetrating trauma. These findings suggest a need for targeted violence intervention programs and improved ED injury surveillance.

Es Factible El Manejo Toracoscopico Con Ventana Positiva En Trauma Penetrante Cardiaco. Diego F Caycedo, Juliana M Ordoñez, Daniel G Nuñez, Tabata L Tinoco, Carlos Alberto, David Giraldo, Mauricio Pedraza, Luis F Cabrera. From the Hospital Universitario Del Valle Evaristo Garcia, Cali, Colombia.

Introducción: Después de la introducción de la ventana pericárdica subxifoidea en 1977, el manejo de los pacientes con trauma cardiaco se ha simplificado, siendo la toracotomía y exploración cardiaca el paso estandarizado mundialmente a seguir cuando la ventana es positiva. En la actualidad el E-FAST ha permitido la selección de los pacientes candidatos a ventana pericárdica. Con la llegada de nuevas tecnologías el abordaje toracoscopico es una opción segura y efectiva, necesitando solo de un personal entrenado. Materiales y métodos: Objetivo: Describir la técnica de realización del abordaje mínimamente invasivo por toracoscopia para ventana pericárdica en pacientes hemodinamicamente estables. Materiales Y Métodos: Paciente ingresado con herida penetrante precordial al servicio de trauma del Hospital Universitario del Valle Evaristo García; revisión historia clínica, y literatura. Resultados: Paciente ingresa por herida por arma corto punzante
4EI con LAA con E-FAST+ subxifoidea; hemoneumotorax izquierdo; toracostomia cerrada drenaje de $200 \mathrm{cc}$; posterior ventana pericárdica por toracoscopia, la cual es positiva con identificación de lesión en pericardio, ventrículo y aurícula izquierdos no sangrantes, hemotórax coagulado residual. Lavado de saco pericárdico con SSN 500 cc hasta obtener líquido claro, aspiración de coágulos. Drenaje de hemotórax y toracostomia. 72 horas posterior retiro de tubo de tórax y egreso sin complicaciones. Conclusiones: El advenimiento de nuevas tecnologías ha permitido simplicar los procedimientos. La cirugía mínimamente invasiva en trauma está siendo una alternativa en pacientes seleccionados con resultados favorables, disminuyendo la necesidad de cirugías abiertas complejas que suelen ser más mórbidas.

Perforación De Divertículo De Meckel Secundario a Trauma Abdominal Por Accidente Automovilístico: Revisión De Un Caso. Sebastián Soto, María T König, Francisca Aliaga, Mónica Martinez. From the Hospital de Urgencia Asistencia Pública, Santiago, Chile.

Introducción:ElDivertículo deMeckel(DM)eslaanomalía congénita más frecuente del sistema digestivo. Aunque generalmente es asintomático, puede presentarse como hemorragia digestiva u obstrucción intestinal. La perforación es infrecuente, asociándose principalmente a cuerpos extraños. Se presenta caso de perforación de DM secundario a trauma abdominal cerrado por accidente automovilístico. Materiales y métodos: Paciente masculino 26 años, ingresa a urgencias tras accidente automovilístico, hemodinamicamente estable. Tras 24 horas, evoluciona con intenso dolor abdominal y deposiciones sanguinolentas, en laparotomía exploradora se evidencia DM perforado. Resultados: Tras 24 horas de hospitalización y dado nueva clínica del paciente, se solicitaTAC de Abdomeny Pelvis con contraste, que describe hallazgos compatibles con probable perforación de víscera hueca, hemoperitoneo y neumoperitoneo leve. Con estos hallazgos se decide ingreso a pabellón de urgencia. Se realiza laparotomía exploradora, donde se evidencia DM perforado y 200 cc de hemoperitoneo, sin observar daño ni signos de perforación en otras vísceras. Se realiza resección del segmento y anastomosis latero-lateral, sin incidentes. El paciente evoluciona favorablemente, por lo que se indica alta al $7 \mathrm{mo}$ día. Se describe en la literatura que la perforación del DM es excepcional y que generalmente se asocia a cuerpos extraños. En todos los casos el cuadro clínico fue inespecífico, caracterizado principalmente por el dolor abdominal intenso y la hemorragia digestiva baja. En ninguna de las publicaciones se realiza un diagnóstico pre operatorio del DM, y nuestro caso clínico no fue la excepción. El estudio de imágenes pre operatorio sólo advierte algunos signos sugerentes de perforación de víscera hueca, por lo que el diagnóstico definitivo se establece en el pabellón y de manera incidental tal como sucedió en este caso. Conclusiones: El DM es una anomalía frecuente del tracto digestivo alto pero sus complicaciones son infrecuentes. Probablemente, existan casos similares al descrito, que no han sido reportados y sería interesante conocer.

Portal Triad Injuries: A National Trauma Data Bank Review. Alejandro Betancourt, Micheal Amaturo, Lisa Szydziak, Sally Kuzniewski, Michael, Jay Yelon. From the Southside Hospital, Northwell Health, Bayshore, New York, USA.

Introduction: Portal triad injuries are infrequent and carry a significant mortality. Little is known about predictors of 
mortality and impact of treatment options. We set to reviewed the National Trauma Data Base Research Data Set 2016 to better understand the incidence, management, predictors of mortality and outcomes associated with portal triad injuries. Materials and methods: We performed a one-year retrospective review of the NTDB-RDS for 2016. The procedures codes for open intervention of the portal triad components were used to identify injuries requiring open operative intervention. Injury description, intervention and mortality were evaluated. Data were analyzed using Chi square and fisher's exact test. A logistic regression model predicting death by interventions adjusting for injury types and severity of injury in other anatomic regions was derived. Results: Of 772,416 patients; a total of 190 underwent open portal trial injury operations. $61 \%$ were penetrating, of which, $89 \%$ were by firearm. Seventy-six percent of the patients were male. $87 \%$ were younger than 55 years old. The injuries and intervention. The overall mortality was $34 \%$ with no difference in mortality when comparing penetrating and blunt mechanisms $(p=0.627)$. Overall mortality was not statistically different when comparing structure injured and type of intervention $(p=0.35)$. In regression analysis, portal vein injuries inferred a higher mortality ( $p=0.0093)$, whereas primary repair of any injury had a survival benefit. Significant concomitant extra-portal injury increased mortality. Finally, when comparing individual anatomic structures injured isolated portal vein Injuries has an odds ratio of death that is four times higher than isolated hepatic artery injuries, controlling for intervention, and severe injury in another region. Conclusion: Portal triad injuries are rare and infer a high mortality. Mechanism of injury has no impact on outcome. Injury structure, the presence of associated significant injury in other anatomic areas and type of intervention were all significant predictors of mortality.

Tratamiento Operatorio vs No Operatorio Del Trauma Hepático, Esplénico O Renal. Resultados Del Registro De Trauma De Fundación Trauma En 14 Hospitales De La Provincia De Buenos Aires. Roberto Klappenbach, Erick Salas, Rafael B Daza, Jorge Suarez, Marilina. From the Hospital Simplemente Evita, González Catán, Buenos Aires, Argentina.

Introducción: El tratamiento no operatorio (TNO) del trauma hepático, esplénico o renal se ha asociado a una disminución de la mortalidad y la morbilidad, aunque se desconoce su uso y sus resultados en nuestra región. El objetivo fue evaluar la tasa de TNO del trauma hepático, esplénico o renal, y su asociación con mortalidad, complicaciones y estadía hospitalaria. Materiales y métodos: Estudio retrospectivo utilizando datos del registro de trauma de 14 hospitales de la red de Fundación Trauma de la Provincia de Buenos Aires, Argentina. Se incluyeron los casos con lesión hepática, esplénica y/o renal, desde abril 2010 a julio 2018. Se consideró TNO cuando no se realizó una laparotomía dentro de las 24 horas del ingreso. Para el análisis se realizó un análisis multivariable, con imputación múltiple para el manejo de datos perdidos, considerando como significativa una p. Resultados: De 637 casos con trauma hepático, esplénico y/o renal, 322 (50,5\%) fueron manejados con TNO. El grupo TNO tuvo menos trauma abierto ( $43 \%$ vs $63 \%$ ), lesiones hepáticas ( $48 \%$ vs $58 \%)$, lesiones esplénicas grado -3 (17\% vs $25 \%)$, alguna lesión hepática, esplénica o renal grado-3 (35\% vs $45 \%$ ) y lesiones abdominales asociadas ( $14 \%$ vs $27 \%$ ), y tuvo más lesiones renales
( $27 \%$ vs $19 \%$ ) y más lesiones de SNC grado-3 (14\% vs $8 \%$ ). En el análisis multivariable, el TNO no se asoció a una reducción significativa de la mortalidad (TNO 18,6\% vs laparotomía 21,3\%; OR 0,76; IC95\% 0,5 a 1,2) ni de la estadía hospitalaria (-2,5 días; IC95\% -6 a 1 día), aunque sí a menos complicaciones (41\% vs 51\%; OR 0,58; IC95\% 0,4 a 0,84), tanto generales como abdominales. Conclusiones: En nuestro contexto, el TNO disminuiría las complicaciones sin modificar la mortalidad ni la estadía hospitalaria. Por las limitaciones del diseño del estudio se necesitan más trabajos para confirmar estos resultados.

Manejo Actual Absceso Hepático En Un Hospital De I Nivel Por El Cirujano De Emergencias. Wilmer FB Capera, Rolando M Rojas, Neftali V Polania, Juan FS Marin, Nohora, Yesid Y Quintero, Gabriela Evers, Luis AR Charry, Gustavo P Perdomo, Francy HS Contreras. From the Universidad Surcolombiana Y Hospital Universitario De Neiva, Huila/Neiva, Colombia.

Introducción: El manejo actual del absceso hepático es el drenaje percutáneo mediante el implante de un catéter multipropósito (pig tail) con guía ecografíca mas la terapia de antibiótico especifica. El absceso hepático es poco frecuente pero tiene alta morbilidad cuando no es diagnosticado y tratado adecuadamente. Objetivo: Presentar los resultados del drenaje percutáneo del absceso hepático un hospital de I nivel en Colombia por cirugía de emergencias. Materiales y métodos: Se realizó en un hospital de I nivel en Colombia, un estudio descriptivo, observacional, retrospectivo, tipo de serie de casos de pacientes con absceso hepático y realización de drenaje percutáneo mediante el implante de un catéter multi propósito (pig tail) con guia por ecografía. La información se archiva en una hoja de cálculo de MS Excel- y se analiza con el programa Epi Info v 7. Resultados: En un hospital de I nivel en el sur de Colombia entre el 2016 y 2017, el diagnóstico de absceso hepático en 18 pacientes tienen dolor en hipocondrio derecho $(94 \%)$, se realizo drenajes percutáneos mediante el implante de un catéter multi propósito con guia por ecografía . El 83.3\% masculino, edad fue 58 años (DS 8 años). El diagnostico $83.3 \%$ ecográfico y $94 \%$ tomografíco. Colección en lóbulo derecho (88.8\%), diámetro mayor $90 \mathrm{~mm}(\mathrm{RI}=82-120$ $\mathrm{mm}$ ). complicaciones (3 casos) así: re implante de catéter 2 casos y desplazamiento 1 caso. No se realizo cirugía abierta. Se presenta 1 caso de mortalidad, que no es asociada a la patología yal drenaje percutáneo. Conclusiones: El absceso hepático poco frecuente, es una patología diagnósticada por ecografia o tomografía. El tratamiento es drenaje percutáneo por catéter multipropósito con guia ecografíca mas antibiótico especifico. En el drenaje percutáneo no hay perforación intestinal, no morbilidad ni mortalidad. Actualmente es mejor que la cirugía abierta.

Características Y Desenlaces Clínicos De Pacientes Con Diagnóstico De Peritonitis Sometidos a Laparotomía De Emergencia En Un Hospital Universitario De Latinoamérica. María F Martínez, Juan P Villegas, Claudia P Orlas, Juan J Meléndez, $J$ José, Juan J Martínez, Kimberly Alcázar, María J Franco, Michael W Parra, Carlos A Ordoñez. From the Fundación Valle del Lili, Cali, Colombia.

Introducción: La cirugía de cuidado agudo ha venido expandiendo su campo dentro del manejo de patologías intra-abdominales médicas y post-quirúrgicas graves, aunque las primeras han sido las más 
estudiadas, la peritonitis post-operatoria cada vez es más frecuente. El objetivo de este estudio es describir las características y desenlaces clínicos de los pacientes sometidos a laparotomía de emergencia en un hospital universitario de alto volumen en Latinoamérica. Materiales y métodos: Todos los pacientes mayores de 18 años, que fueron sometidos a laparotomía de emergencia entre Enero/2012-Diciembre/2018 fueron incluidos. Se clasificaron así: peritonitis médica (grupo I) y peritonitis post-quirúrgica (grupo II). Todas las laparotomías por trauma fueron excluidas. Resultados: 198 pacientes fueron incluidos, 100 (50.5\%) con peritonitis médica y $98(49.5 \%)$ con peritonitis post-quirúrgica, la mayoría de los pacientes estaban hemodinámicamente estables al ingreso [grupo I = PAS: 113 mm Hg (IQR: 96-133), FC: 97 Ipm (IQR: 83-111), grupo II = PAS: 115 mm Hg (IQR: 100-130) FC: 89 Ipm (IQR: 80-110)], hubo mayor proporción de cáncer en los pacientes del grupo II [grupo I = 27 (27\%), grupo II = 41 (41.8\%; $p=0.03$ y, la mayoría de los pacientes fueron clasificados como ASA-2 [46 (23.2\%)] y ASA-3 [84 (42.4\%)]. Los pacientes del grupo II requirieron de manera significativa más estancia en $\mathrm{UCl}$, estancia hospitalaria y la proporción de mortalidad fue mayor. Conclusiones: En nuestro estudio hubo una proporción alta de pacientes que fueron diagnosticados con peritonitis postquirúrgica, ellos requirieron más días de UCl, estancia hospitalaria y tuvieron una proporción significativa de mortalidad cuando se compararon con los pacientes con peritonitis de origen médico.

Gravidade Do Trauma E Lesões Associadas Ao Uso De Patinetes Elétricos. Genesis Barbosa, Ane K Bonfim, Regina Sousa, Lilia Nogueira. From the Universidade de São Paulo, São Paulo, Brazil.

Introducción:Os patinetes elétricos para locaçãoe compartilhamento representam um meio de transporte rápido e fácil para curtas distâncias e, nos últimos anos, esses dispositivos foram rapidamente difundidos em grandes centros do mundo. As lesões decorrentes do uso de patinetes são novo fenômeno; entretanto, a incidência e os tipos de lesões associadas a esses equipamentos são desconhecidos. Neste sentido, este estudo teve por objetivo caracterizar as lesões, a gravidade do trauma e a evolução clínica de pacientes com traumatismos associados ao uso de patinetes elétricos. Materiales y métodos: Coorte prospectiva de pacientes que sofreram trauma decorrente do uso de patinetes admitidos, entre dezembro/2018 e abril/2019, em serviço de emergência hospitalar de um hospital no Rio de Janeiro, Brasil. Utilizou-se a Abbreviated Injury Scale para mapear a gravidade das lesões e os índices revised trauma score (RTS), injury severity score (ISS) e trauma and injury severity score (TRISS) para mensurar a gravidade do trauma. Estatísticas descritivas foram realizadas para caracterizar a amostra. Resultados: Um total de 28 pacientes (67,9\% sexo masculino, idade média 29,18 \pm 8,07 anos) foram admitidos no serviço de emergência após trauma decorrente do uso de patinete. Desses, $82,1 \%$ eram ocupantes do patinete e $7,1 \%$ relataram o uso de capacete. Quanto à gravidade do trauma, as médias do RTS, ISS e TRISS foram 7,39 \pm 0,66, 8,61 $\pm 3,67$ e 98,96 $\pm 0,83$, respectivamente. Superfície externa $(75,0 \%)$, cabeça/pescoço $(28,6 \%)$ e tórax $(28,6 \%)$ foram as regiões corpóreas mais acometidas e, em média, os pacientes apresentaram 2,14 regiões lesionadas. Houve necessidade de cirurgia em $32,1 \%$ da casuística, sendo que a maioria dos pacientes foi internada $(64,2 \%)$ e dois $(7,1 \%)$ morreram.
Conclusiones: $\mathrm{O}$ uso de patinetes elétricos tem sido crescente, assim como a ocorrência de lesões associadas, algumas com elevada gravidade que resultaram em óbito. Esses resultados reforçam a necessidade de medidas preventivas que fortaleçam o uso consciente desse meio de transporte.

Evaluacion Tomografica Del Trauma Cerrado. Laura JD Mateus, Luis FC Tavera, Maria FJ Garcia, Laura IV Angel, Juan Pablo, Andrea JP Camacho, Silvia B Serrano. From the Hospital Universitario de Santander, Santander/Bucaramanga, Colombia.

Introducción: El trauma es la sexta causa de muerte a nivel mundial. La localización toracoabdominal es frecuente, y la sobrevida depende del diagnóstico y tratamiento oportuno. La tomografía multicorte es el estudio diagnóstico más sensible en lesiones toracoabdominales; sin embargo, ocasionalmente está disponible de inmediato, pero su reporte por radiólogo puede retrasarse, teniendo el cirujano la responsabilidad de tomar decisiones con su interpretación de las imágenes. Nuestro objetivo es establecer la concordancia interobservador (radiólogo vs cirujano) en la evaluación tomográfica del trauma cerrado toracoabdominal en un hospital de tercer nivel. Materiales y métodos: Se realizó un estudio de cohorte durante 2 años, incluyendo pacientes ingresados a urgencias del hospital universitario de Santander con trauma de toracoabdominal cerrado. Se obtuvieron las tomografías contrastadas realizadas al ingreso y se interpretaron por ambos observadores usando el software Vítrea. Los datos recolectados se analizaron en Stata 11, calculando el índice kappa $(\kappa)$ como medida de acuerdo interobservador. Se estableció $\kappa>0.6$ como aceptable, $>0.8$ moderado y 1.0 casi perfecto. Resultados: El 90\% de la población fue género masculino, con edad promedio de 39 años. El mecanismo más frecuente fue accidente de tránsito en $71 \%$, seguido por caída $17 \%$. Se analizaron el $100 \%$ de las interpretaciones, encontrando un k en lesión pulmonar de 0.63 , esplénica 0.26 , hepática 0.36 , neumoperitoneo 0.00 y liquido libre intraabdominal 0.00 . Conclusiones: El acuerdo interobservador es aceptable en el diagnostico tomográfico de trauma pulmonar por los cirujanos en comparación con los radiólogos. La concordancia en el diagnóstico de las demás lesiones es inaceptable, siendo los casos concordantes posiblemente por azar. Es evidente la importancia de la disponibilidad de radiología como parte del grupo multidisciplinario en atención de trauma y la relevancia del entrenamiento en la evaluación tomográfica del trauma cerrado para los futuros cirujanos.

Experiencia En El Manejo De Heridas Por Arma De Fuego Transpelvicas. Jesus Lugo, Pablo Ottolino, Analia Zinco, Juan P Ramos, Claudia, Jose Ruiz, Carolina Munoz. From the Hospital Dr Sotero del Rio, Santiago, Chile.

Introducción: Las heridas por arma de fuego en pelvis requieren particular atención debido a la alta densidad de órganos contenidos dentro de la pelvis ósea, presentando alta tasa de morbi-mortalidad. Objetivo: Describir las características demográficas, así como el diagnóstico, manejo y complicaciones de los pacientes que ingresan con trauma pélvico penetrante por arma de fuego. Materiales y métodos: Estudio transversal, descriptivo, en el que se incluyen pacientes mayores de 15 años ingresados al Hospital 
Dr Sótero del Río (Chile) y Hospital Dr Domingo Luciani (Venezuela) con el diagnóstico de trauma pélvico penetrante por arma de fuego en un período de 3 años, de enero del 2015 a enero del 2018. Resultados: Serie compuesta por 94 pacientes con trauma pélvico penetrante por arma de fuego. Doce pacientes (13\%) ingresaron con PAS/PAD menor de $90 / 60 \mathrm{mmHmg}$, constatando 26 pacientes (27\%) con frecuencia cardiaca al ingreso mayor a 100 latidos por minuto. EI FAST estuvo Negativo en 91 casos (97\%). Dentro de los estudios diagnósticos predominó: Tomografía (30\%), radiografías (11\%), Doppler (7\%), rectosigmoidoscopia (5\%), uretrocistografia (4\%). Las laparotomias terapéuticas fueron en 56 casos (73\%) y no terapéuticas en 9 (12\%). Organos mas frecuentemente lesionados: asa delgada (36\%), genitales (6\%), colon (5\%), pelvis (4\%), recto (3\%) y vejiga (2\%). Se realizó cirugía control de daños en 8 pacientes (9\%). El 5\% de los pacientes requirieron mas de 10 uds de Concentrado globular. 68 pacientes (73\%) requirió menos de 7 días de hospitalización. La complicaciones mas frecuentes fueron: Infección de herida operatoria (8\%), íleo (3\%), hematoma de herida (2\%). La Mortalidad fue de $3 \%$. Conclusiones: El trauma pélvico penetrante por arma de fuego representa un reto para el cirujano de urgencia. Pacientes en shock tienen alta incidencia de trauma vascular. Los pacientes estables se benefician de una adecuada evaluación diagnóstica inicial.

Exactitud Diagnóstica En Tomografía De Trauma De Torso: ¿Está El Cirujano De Emergencia En Capacidad De Interpretar Las Imágenes Sin Necesidad Del Radiólogo? Analia Zinco, Pablo Ottolino, Juan P Ramos, Jose ER Cabrera, Jesus Alejandro, Claudia Arenas, Carolina M Alarcon. From the Unidad De Trauma Y Urgencias, Hospital Dr Sótero Del Río, Santiago, Chile.

Introducción: En las últimas décadas, se ha incrementado el uso de la tomografía computarizada (TC) en la evaluación inicial del paciente politraumatizado, la cual ha demostrado aumento en la supervivencia, menor tiempo de hospitalización y menor tasa de lesiones desapercibidas. El objetivo es determinar la aproximación diagnóstica de los hallazgos encontrados por el cirujano de emergencia en la TC del paciente con trauma de torso, y la comparación con un radiólogo experto. Materiales y métodos: Estudio observacional, analítico, que incluye 30 tomografías de pacientes con trauma de torso ya sea penetrante o contuso, las cuales fueron interpretadas de forma aleatoria por 100 cirujanos y residentes de cirugía y posteriormente comparadas con el análisis realizado por el radiólogo. Resultados: La serie incluye 100 participantes (residentes de cirugía o cirujanos), de los cuales $85 \%$ son cirujanos. La discrepancia total entre los hallazgos de los participantes y el radiólogo fue de 11,6\% para los residentes de cirugía y 5,4\% para los cirujanos. Sin embargo esta varía según órgano evaluado, encontrándose que en abdomen, el hígado, bazo, riñón y hemoperitoneo, fueron los hallazgos con menos discrepancias; mientras que lesión de víscera hueca fue el hallazgo menos identificado. En tórax, la menor discrepancia se encontró en la identificación de hemotórax, neumotórax, contusión pulmonar y enfisema subcutáneo; mientras que los hallazgos menos identificados fueron fracturas costales y vertebrales, los cuales no cambiaron la conducta a realizar por el cirujano general. Conclusiones: La tomografía computarizada es una alternativa válida para el diagnóstico de lesiones del paciente politraumatizado grave durante la evaluación inicial, la cual puede ser interpretada de forma acertada por el cirujano de emergencias para la toma rápida de decisiones, sin necesitar de un radiólogo en este punto del nivel de atención.

Epidemiological Profile of Eye Trauma in a Reference Service in São Luís: Maranhão. Daniel De Brito Pontes, Vitor F Gerude, Paulo HS Bezerra, Felipe AC Santos, Raylenne M Dos, Luana B Ribeiro, Clara A De Alencar, Luana N Gonçalves. From the UNICEUMA, Maranhão/São Luís, Brazil.

Introduction: Trauma is a major cause of ocular morbidity, especially in children and young adults. Its occurrence translates into strong socioeconomic impacts, as well as on the quality of life of the affected patients. It is known that trauma is the leading cause of preventable blindness in the world, with about one-third of cases of unilateral visual impairment. Materials and methods: The present study was carried out with the purpose of evaluating the epidemiological characteristics of patients attending a referral service in São Luís do Maranhão for mechanical ocular trauma from March 2016 to February 2017. This is a crosssectional observational descriptive study. Epidemiological data of interest were collected through a standardized service record with subsequent analysis of the associated frequencies. A total of 154 patients were treated during the study period, of whom 99 (64.3\%) due to closed ocular trauma and 55 (35.7\%) due to open trauma. Results: The general characteristics were: mean age of 30.1 years, predominantly the age group of under 21 (36.4\%), male (81.8\%) and residents of the interior of the state (55.2\%). Regarding the occupation of the patient, it was observed that students or dependents were the majority (34\%) and among those who worked, the highest frequency was of farmers (19\%). The place of the trauma occurred predominantly at home (38.9\%), followed by work (29.9\%). Of these, a minority used personal protective equipment at the time of injury (6.5\%). The main mechanisms of trauma were puncture-sharp objects (22.1\%), vegetal materials (21.4\%) and blunt objects (20.1\%). Conclusions: Therefore, the importance of the development and implementation of strategies for the prevention of accidents, both at home and at work, is reinforced. In addition, the importance of the creation of measures that promote eye health in primary care is highlighted, with the purpose of reducing the incidence of preventable visual impairment.

Utilidad De La Tomografía Axial Computarizada En El Diagnóstico De Lesiones Diafragmáticas Penetrantes En Pacientes Estables. Bryan SU Trochez, Gabriel FD Cajas, Fabián Valdés, Mónica Morales, Manuel, Ana M Del Valle. From the Universidad Del Valle, Cali, Colombia.

Introducción: Cuando se presenta trauma toracoabdominal penetrante existe una alta probabilidad de presentar heridas diafragmáticas. En la mayoría de los casos se requiere la realización de la intervención quirúrgica como medida diagnóstica, por ello surge la Tomografía como una herramienta que puede ser de ayuda en el diagnóstico no invasivo de estas heridas. Materiales y métodos: El objetivo de este proyecto es evaluar el rendimiento de la Tomografía en el diagnóstico de heridas diafragmáticas en pacientes con trauma toracoabdominal penetrante que se encuentran estables. Es un estudio de concordancia, se hace una descripción sociodemográfica de la muestra 
y se hace una validación de pruebas diagnósticas donde se compara la Tomografía como un método alternativo contra los hallazgos quirúrgicos. Realizado en el Hospital Universitario del Valle en Cali-Colombia entre Marzo de 2012 y Junio de 2015. Resultados: Muestra de 119 paciente elegibles, en su mayoría hombres $(90,7 \%)$, con edad promedio de 23 años. Se obtuvo una sensibilidad del $94.4 \%$, especificidad del $46.8 \%$, con un valor predictivo positivo (VPP) de $44.7 \%$ y valor predictivo negativo (VPN) de 94.8\%. También se estimó el Likelihood Ratio positivo $(\mathrm{LR}+)$ de 1.78 y el negativo (LR-) 0.12 y coeficiente de $\kappa 0.4425$. Conclusiones: Se encontraron resultados que muestran diferencias con lo esperado, que estaba fundamentado en la literatura disponible, pero la mayor parte de la evidencia disponible son estudios retrospectivos. Se trata de un estudio muy sensible pero poco específico, pero con un alto valor predictivo negativo (94\%) lo que sugiere puede anexarse dentro de los algoritmos diagnósticos para los cuales es una buena herramienta como parte del manejo conservador de los pacientes. Es ideal avanzar en homogeneizar las estrategias de interpretación de la Tomografía dada la concordancia moderada encontrada entre los lectores $(\kappa: 0,44)$.

Uso De La Tomografia Corporal Total Durante La Evaluación Inicial De Los Pacientes Con Trauma Severo: Es Posible? Luisa Ortiz, Diego Beltrán, Claudia P Orlas, Erika Rincón, Natalia, Alfonso Holguín, Carlos A García, Alberto F García, Michael W Parra, Carlos A Ordoñez. From the Fundación Valle Del Lili, Cali, Colombia.

Introducción: La Tomografía Corporal Total es una herramienta novedosa, sin embargo, su uso en algunos escenarios continúa siendo controversial. El objetivo de este estudio fue determinar si es factible el uso de la Tomografía (TAC) Corporal Total durante la evaluación inicial de los pacientes con trauma cerrado (TC) y penetrante (TP) severo. Materiales y métodos: Todos los pacientes mayores de 15 años, que durante su ingreso requirieron la activación del código de trauma y se les realizó TAC corporal total, en un Centro de Trauma Nivel I, entre 2017-2018 fueron incluidos. Las variables sociodemográficas, estado de ingreso, y desenlaces clínicos fueron reportadas. Resultados: Se incluyeron 263 pacientes, la mayoría con trauma cerrado [218 (82.9\%)], un cuarto de los pacientes ingresaron hemodinámicamente inestables [65 (25\%)] y, se reportó un puntaje de Glasgow significativamente más bajo entre ellos [TC: Glasgow: 12 (IQR: 7-15), TP: Glasgow: 15 (IQR: 10-15); $p=0.01]$. A $172(65 \%)$ se les realizó Manejo Selectivo No Operatorio luego de la evaluación con la imagen, la mediana de tiempo entre el ingreso a urgencias y la toma del TAC fue de 29 minutos (IQR: 14-55), y el tiempo de diagnóstico luego de tomada la imagen fue similar entre ambos grupos [TC: 22 min (IQR: 13-33) vs TP: 23 (IQR: 14-28); $p=0.96]$. No hubo diferencias estadísticamente significativas en los niveles de radiación y mortalidad entre los subgrupos. Conclusiones: El uso de la TAC corporal total durante la evaluación inicial de los pacientes con trauma cerrado y penetrante severo es factible, incluso en pacientes con inestabilidad hemodinámica, en un margen de tiempo adecuado, que permite clasificar correctamente los pacientes que no se benefician de una intervención quirúrgica.

Experiencia En Resucitación Con Sangre Entera Fresca, Logística Y Resultados. Juan CS Molina, Jeovanni R Naranjo, Raul P Andrade, Lenin F De Córdova, Hernan, Nube Flores, Cecibel Cevallos, Juan C Puyana. From the Hospital Vicente Corral Moscoso, Azuay/ Cuenca, Ecuador.
Introducción: En la última década el manejo del trauma severo ha cambiado de cirugía de control de daños a a nueva estrategia resucitación en control de daños; la resucitación hemostática con hemocomponentes en una relación 1:1:1 ha demostrado ser más efectiva, pero no es aplicable en muchos hospitales Latinoamericanos, así presentamos la propuesta de sangre entera fresca (SEF), logística y resultados. Materiales y métodos: La donación es el mecanismo para la obtención de sangre, el banco determina la idoneidad, confidencialidad, extracción y reinfusión. Disponemos de protocolos para disponer de 5 unidades de SEF. Presentamos un estudio prospectivo noviembre de 2012 hasta febrero de 2019. Resultados: Presentamos 70 pacientes, ISS $25=29$, masculinos 63 (90\%), edad media 35 años (7-92), mediana 28 y moda 20, 21-20 años $40 \%$, región urbana 46 (71\%), arma blanca 40 (57\%), accidente de tránsito 19 (27\%), caídas 8 (11\%), arma de fuego 3 (5\%). Tiempo hasta ingresar al quirófano $52 \mathrm{~min}$; cristaloides (emergencias + quirófano) 2.8 L. PAM 67.20 ( $\pm 19,24)$; frecuencia cardíaca 104,97 $( \pm 20,95)$; respiraciones $23,45( \pm 5,33)$; temperatura 36,03 OC $( \pm 0,48)$; días de hospitalización 6,10 días $\pm 5,26$. Promedio de la resucitación con SEF utilizó 2 unidades de SEF y 2 unidades de GR y 2 unidades de plasma fresco congelado. Existió 1 caso de mortalidad. Conclusiones: La resucitación con SEF es factible y es una muy buena alternativa en nuestros países donde es difícil disponer del 1:1:1 y los resultados en el escenario de RCD la resucitación con SEF es igual de efectiva.

Axillary Artery Exploration in Trauma: Description of an Alternative Approach. Juliana M Ordoñez, Carlos A Ordoñez, Daniel G Nuñez, Diego FC Medina, Mauricio, David Giraldo. From the Fundacion Valle Del Lili, Cali, Colombia.

Introduction: Axillary artery injuries are rare, usually associated with shoulder dislocation, fractures of the proximal humerus and penetrating trauma. Examination can be technically demanding due to the anatomical position of the vessel and associated concomitant injuries. All patients with axillary or periclavicular trauma should be evaluated for vascular and brachial plexus trauma. Angiography is reserved for stable patients with soft signs of vascular trauma and very rarely for the stable patient with hard signs without a clear site of injury. Materials and methods: Objectives: Description of an alternative technique for axillary artery exploration in trauma materials literature review and case/technique description detailed photographic documentation with an overview of technical aspects of dissection and vascular repair. Results: The anterior approach on the deltopectoral groove for an exploration just after the third portion of the subclavian is the classic indication, however, in this case report, a patient with a projectile injury from a gun in the thoracic region with an ipsilateral suprascapular exit wound is described. On admission to the emergency department the patient is hemodynamically stable, with an angiography revealing an axillary artery lesion. In the operating room an axillary approach similar to the one performed for IV lymphadenectomy given the adequate exposure. A 50\% circumference lesion was identified in the middle third and a Gore-Tex graft anastomoses with adequate perfusion was performed, presenting an uneventful postoperative. Conclusions: Axillary artery injuries are rare even in penetrating 
wounds although the index of suspicion must be high. The anterior classic approach may present technical limitations so the described axillary approach could facilitate dissection and adequate repair of these lesions.

Consumo De Alcohol, Relación Con El Trauma, Su Severidad Y Mortalidad. Santiago Dardanelli, Edward Delgado, Marcelo Mion, Julio Trostchansky, Fernando, Paula Morgade. From the Hospital De Clínicas, Montevideo, Uruguay.

Introducción: El alcohol es uno de los factores de riesgo más importantes para sufrir una lesión grave. Evaluar la frecuencia y el impacto del uso indebido de alcohol sobre la generación y gravedad del trauma es necesario para apoyar políticas destinadas a disminuir el consumo. Hemos detectado un vacío de información estadístico de la cantidad de traumatizados y la vinculación de estos con el consumo de alcohol. Se planteó estudiar la frecuencia del consumo de alcohol en el trauma por siniestros de tránsito y vincularlo con la gravedad y mortalidad del mismo. Es un verdadero problema de salud pública. Materiales y métodos: Objetivos: Estudiar la asociación existente entre el consumo y grado de consumo de alcohol y la severidad y mortalidad del trauma. Implementar medidas de prevención. Estudio transversal. Población: Pacientes traumatizados asistidos en el Departamento de emergencia del Hospital de Clínicas, entre Marzo Agosto 2017. Variables: Sexo, Edad, ocupación, Evento traumático (Tipo, Topografía, Severidad del trauma (ISS), Consumo de Alcohol (AUDIT), Alcoholemia. Resultados: Se incluyeron 121 casos. La mayoría correspondió a traumas cerrados (86\%), y dentro de éstos al siniestro de tránsito. Si analizamos los traumas penetrantes, que constituyeron el $14 \%$ del total. La mayoría de las alcoholemias fueron negativas (72\%); las alcoholemias positivas correspondieron al $28 \%$ de la serie (34 casos). De acuerdo al ISS, la cantidad de traumas severos correspondió al $27 \%$. Se obtuvo asociación estadisticamente significativa entre las alcoholemias positivas y severidad del trauma. No se encontró correlación entre el nivel de alcoholemia y la severidad del trauma medida por la escala ISS. Conclusiones: El consumo de alcohol se asocia significativamente con la severidad del trauma. No encontramos asociación entre los niveles de consumo de alcohol y la severidad en el trauma. La mortalidad también aumenta frente al consumo de alcohol.

True-Bolivia (Trauma Responders Unify to Empower Communities) in Santa Cruz, Bolivia: Participant Baseline Characteristics and Opinions. Erica K Ludi, Pablo Peñaranda, Leah Tatebe, Esteban F Gutierrez, Mamta. From the Northwestern University, Santa Cruz, Bolivia.

Introducción: More than half of all trauma deaths occur in the pre-hospital setting with low- and middle-income countries (LMICS) shouldering the largest burden. Coordinated prehospital network implementation, including first responder and ambulance driver skill trainings, can reduce mortality. First responder courses in Bolivia have improved participant knowledge and confidence levels. The current study aims to analyze participant baseline characteristics and post-workshop evaluations to inform future course promotion and development. Materiales y métodos: TRUE-Bolivia is a 4-hour didactic and practical trauma first responder course that covers scene safety, basic airway management, bleeding control, and pelvic binding. Course participants were recruited in Santa Cruz, Bolivia. Participants who completed all pre-and post-course assessments were included. Quantitative data was aggregated in Microsoft Excel, and qualitative data was analyzed for thematic content. Resultados: 27 people participated in three courses. Average age was 28.9 years, and 17/27 (63\%) were female. 9/27 (33\%) were in medical training, $5 / 27$ (18.5\%) did house labor or baby-sitting, while $1 / 27$ (3.7\%) worked in public transportation. 13/19 (68.4\%) admitted to never wearing a seatbelt when in a car, and 12/17 (70.6\%) never wear helmets while on a motorcycle. On the post-course evaluation, $12 / 26$ (46\%) quoted that learning new skills was the most beneficial aspect of the course, $6 / 26$ (23.1\%) quoted helping others, and $8 / 26(30.8 \%)$ stated a combination of the two. Future course improvement suggestions included adding content on burns, CPR, head and neck injuries, and offering more courses in general. Conclusiones: TRUE-Bolivia empowers participants to help others at the scene in a setting where ambulances can take over an hour to arrive. Strategies will be developed to include more public transport workers as they likely arrive first on scene in order to maximize course impact. Course improvement suggestions will be evaluated for appropriateness in order to maintain the focus on trauma prevention and initial management.

Experiencia Del Manejo De Pacientes Con Sepsis Intraabdominal Severa Con Laparostomía Contenida Con Bolsa De Bogotá En El Hospital Luis Vernaza En El Periodo 2014-2018. Wilson S Loor, Jose N Moreno, Marlon SM Riera. From the Hospital Luis Vernaza, Guayaquil, Ecuador.

Introducción: El "abdomen abierto" consiste en la separacion de la pared abdominal con exposición visceral de manera controlada e intencional posterior a una laparotomía. La bolsa de Bogotá se ha descrito para la cobertura temporal del abdomen hasta su cierre definitivo. El objetivo de este trabajo ha sido describir nuestra experiencia. Materiales y métodos: Estudio retrospectivo que incluyo pacientes manejados como laparostomía contenida con bolsa de Bogotá por sepsis intrabdominal en el Hospital Luis Vernaza del 2014 al 2018. Se excluyeron pacientes con etiología nefrourológica o pancreatitis grave. Se revisaron variables demográficas como sexo y edad, estancia hospitalaria, días en $\mathrm{UCl}$, patología de ingreso, indicación de laparostomía contenida, numero de lavados realizados y morbimortalidad asociadas. Resultados: Se identificaron 73 pacientes manejados con bolsa de Bogotá, 61 (84\%) fueron hombres; el grupo etario con mayor número de casos (58) fue entre 15-44 años (79\%). El promedio de estancia hospitalaria fue de 50 días, con un tiempo máximo de 203 días. La etiología de la peritonitis fue postraumática en $24 \%$ de los casos, posquirúrgica por trauma en $26 \%$, perforativa en $25 \%$, postoperatoria por patología aguda (POPA) en $12 \%$ y $8 \%$ por otras causas. El número promedio de relaparotomías fue de 6 . La complicacion más frecuente fue el shock séptico (51\%). La mortalidad general fue de 16 pacientes (22\%), 6 (38\%) del grupo de peritonitis postoperatoria por trauma, 6 por perforacion no traumática (38\%), 1 (13\%) por peritonitis postoperatoria por patología aguda (POPA) y 2 (25\%) por peritonitis traumática. El cierre definitivo fue posible en 32 pacientes 
(43\%); los 15 restantes quedaron con "hernia planeada" (21\%). Conclusiones: La bolsa de Bogotá es un método útil para el manejo del abdomen abierto por sepsis. Se trata de un material económico, inerte, fácil de colocar y disponible en los centros de cuidados críticos.

Utilizing a Trauma Capacity Assessment to Inform Trauma Center Designations and Pre-hospital System Improvements. Erica K Ludi, Jose C Camacho, Andres Cuellar, Mamta Swaroop, Esteban. From the Northwestern University, Santa Cruz, Bolivia.

Introduction: No coordinated trauma system exists in the Department of Santa Cruz in Bolivia. Injured patients frequently have multiple hospital encounters before arriving at the appropriate level of care needed due to the tiered (primary, secondary, and tertiary), inter-hospital referral system in place. The aim of the current study was to evaluate the trauma capacity of facilities in Santa Cruz in order to inform trauma center designations and prehospital triage protocols. Materials and methods: The PIPES (Personnel, Infrastructure, Procedures, Equipment, Supplies) tool, an internationallyvalidated surgical capacity assessment, was translated into Spanish and modified to include trauma-specific items. Hospitals were evaluated with in-person interviews. Data were tabulated, coded, and analyzed with Microsoft Excel. Results: Two tertiary-level public hospitals, one tertiary-level private hospital, and two secondary-level public hospitals participated in the assessment. The average number of hospital beds is 168 (range 74-250). No fellowship-level trained trauma surgeons exist, but providers with ATLS training range from 3-12 at each hospital. Only $60 \%$ (3/5) of facilities have a general surgeon present in house 24-hours a day, but all have at least one Emergency Medicine physician present continuously. The average number of operating rooms is $5(3-7)$ with $0-1$ available for emergencies at all times. Eighty percent (4/5) have an Intensive Care Unit (range: 4-14 beds). All hospitals had the capacity to perform general surgery procedures, while only $60 \%$ have neurosurgical, thoracic, and burn management surgical capabilities. Conclusion: Capacity to care for trauma patients is limited and unevenly distributed in Bolivia. Identifying facilities that meet a minimal standard will improve prehospital triage, arrival to definitive care, and overall patient outcomes. While the PIPES tool is limited by self-report and specialty of the selected interviewee, assessment results will lead to stronger recommendations regarding training programs and capacity building.

Experiencia En El Tratamiento Del Trauma Vascular Periférico En El Hospital Dr Domingo Luciani. Yovany RC Contreras, Roberto AG Belandria, Luis M Richard, José F Vivas, José León, Pablo Ottolino, Liliher Gonzalez, Willy Neumann. From the Hospital General Del Este Dr Domingo Luciani, Caracas, Venezuela.

Introducción: El trauma vascular periférico es uno de los principales retos quirúrgicos para los cirujanos de trauma y urgencias, su entrenamiento adecuado es esencial debido a la alta frecuencia y complejidad de estos casos. Por ello describimos la epidemiologia, procedimientos quirúrgicos frecuentes y complicaciones en el Hospital General Dr Domingo Luciani. Materiales y métodos: Estudio retrospectivo, transversal correlacional. Se utilizó la base de datos de los servicios de
Cirugía General I y II del Hospital Dr Domingo Luciani, usando palabras para filtrar la información, como: lesión vascular, poplítea, femoral, braquial y axilar, para un total de 48 pacientes. Resultados: El 64\% (40) están entre 15-30 años y 83\% (52) son masculinos. Predominó el trauma penetrante en un $91 \%$ (57). El trauma toracoabdominal representó la mayor lesión asociada $32 \%$ (20), seguido de fracturas en las extremidades $11 \%$ (7). Las lesiones combinadas de arteria y vena fueron frecuentes, en las que se realizó ligadura venosa 50\%, injerto autólogo (safena) 38\% y anastomosis termino-terminal 37\%, como procedimientos reconstructivos arteriales. En 20\% (13) se hicieron fasciotomías profilácticas y $6 \%$ (4) fasciotomías terapéuticas. El 3\% (2) se trataron con amputación de extremidad como procedimiento inicial y $9 \%$ (6) como fracaso de la cirugía inicial. La mitad de las cirugías fueron realizadas por cirujanos generales, el restante por cirujanos vasculares y de trauma. La infección del sitio quirúgico $24 \%$ (15) fue la complicación más frecuente. El 16\% (10) de los pacientes fallecieron por shock hipovolémico y $84 \%$ (52) egresaron por mejoría. Conclusiones: Evitar complicaciones y la muerte de un paciente con trauma vascular periférico es el objetivo principal de todo cirujano, actualmente se tiende a salvar la extremidad más que a amputarla, siendo los cirujanos generales y de urgencias los que deben estar capacitados para realizar los procedimientos quirúrgicos adecuados en el momento preciso.

Evaluación Clínica Objetiva Estructurada (Ecoe) Basado En Simulación Para La Evaluación Y Entrenamiento De Habilidades Quirúrgicas Avanzadas En Trauma. Caterina Contreras, Javier Vela, Cristián Jarry, Pablo Ottolino, Gabriel, Julián Varas, Pablo Achurra. From the Pontificia Universidad Católica De Chile, Santiago, Chile.

Introducción: El manejo quirúrgico adecuado y oportuno tiene alto impacto en mortalidad por trauma. Los cirujanos en formación tienen cada vez menores oportunidades de participar en casos de trauma severo. La simulación permite entrenar y evaluar competencias en forma efectiva, pero faltan modelos adecuados para manejo quirúrgico del trauma. Objetivo: Describir y validar un ECOE para entrenamiento y evaluación de competencias quirúrgicas avanzadas en trauma. Materiales y métodos: Se desarrolló un ECOE con 6 estaciones simuladas usando material ex vivo. (1) Suturas y anudación. (2) Resección intestinal y anastomosis (intestino bovino). (3) Anastomosis vascular termino-terminal (vasos perfundidos de 5 $\mathrm{mm}$ ). (4) Reparación injuria pulmonar (modelo porcino ventilado ex vivo). (5) Reparación injuria cardiaca (corazón porcino perfundido). (6) Sutura laparoscópica de injuria intestinal. Se midieron residentes de segundo (R2), cirujanos recién graduados (CRG) y expertos. Tuvieron 20 minutos por estación para completar el procedimiento. Cada estación contaba con un asistente pasivo. Todos los procedimientos fueron grabados para posterior evaluación ciega por expertos usando escalas generales y específicas validadas (OSATS) con puntaje máximo 25 para cada estación (150 total). Además se midió permeabilidad, filtración y tiempo operatorio. Resultados: Participaron 8 R2, 8 CRG y 3 expertos, obteniendo puntaje OSATS promedio 82, 113 y 147 respectivamente (p). Conclusiones: Un ECOE con estaciones simuladas utilizando tejido ex vivo permite evaluar competencias quirúrgicas avanzadas en trauma en residentes y cirujanos generales. Podría usarse para entrenamiento y perfeccionamiento de técnicas quirúrgicas. 
Programa De Entrenamiento Simulado En Disección Cervical. Caterina Contreras, Javier Vela, Cristián Jarry, Juan P Ramos, Pablo, Julián Varas, Pablo Achurra. From the Pontificia Universidad Católica De Chile, Santiago, Chile.

Introducción: En Chile, el trauma es la primera causa de muerte y discapacidad en menores de 40 años. Los residentes de cirugía tienen cada vez menor exposición a pacientes con trauma grave producto de restricción horaria y preocupación por la seguridad de los pacientes. La simulación ha demostrado reducir las curvas de aprendizaje de procedimientos quirúrgicos y podría mejorar el desempeño de cirujanos recién egresados en el manejo del trauma cervical y abdominal. Objetivo: Desarrollar y evaluar un programa de entrenamiento simulado en disección cervical en modelo porcino ex vivo. Materiales y métodos: Previa aprobación por comité de ética, se enrolaron 8 residentes de cirugía general de $2^{\circ}$ año (R2) y 5 de $3^{\circ}$ año (R3). Debían hacer una cervicotomía, identificar vena yugular externa, arteria carótida y nervio vago; e identificar y controlar esófago. Se utilizaron pautas de cotejo (OSATS general y específico), considerando tiempo operatorio y número de estructuras vitales dañadas durante la disección. Se evaluó el desempeño de 2 expertos en el modelo a modo de comparación y definir estándares de evaluación para el puntaje de corte. Resultados: R2 requirieron en promedio 7 sesiones para alcanzar el puntaje mínimo de aprobación, mientras que R3 requirieron 5 sesiones. OSATS general y específico fue mayor en R3. En la medida que progresaron, los residentes dañaron menos estructuras vitales durante la disección y disminuyeron sus tiempos operatorios. En comparación con expertos, los residentes presentaron en general menor puntaje OSATS general y específico; aunque el grupo R3 no presentó diferencias estadísticamente significativas con los expertos en OSATS específico a la quinta sesión. Todos los alumnos aprobaron la sesión en porcino vivo anestesiado al primer intento. Conclusiones: Un programa estandarizado de disección cervical en modelo porcino eutanasiados es efectivo para entrenar residentes. Esto podría permitir disminuir los costos y problemas éticos asociados al entrenamiento en animales.

Competencias Adquiridas Y Seguridad De Los Cirujanos Chilenos Recién Egresados En El Manejo Del Trauma En Urgencias. Javier Vela, Caterina Contreras, Leonardo Cárcamo, Pablo Achurra, Claudia, Juan P Ramos, Rubén Ávila, Rolando Rebolledo, Julián Varas, Jorge Martínez. From the Pontificia Universidad Católica De Chile, Santiago, Chile.

Introducción: En Chile el trauma constituye la tercera causa de muerte en población general y la primera en población bajo 45 años laboralmente activa. Las competencias y seguridad para realizar estos procedimientos después de la formación del cirujano general no han sido evaluadas previamente en nuestro país. Objetivo: Evaluar competencias y seguridad entregada por los programas de formación en cirugía general de Chile acerca del adecuado manejo del trauma de urgencias. Materiales y métodos: Estudio de corte transversal y descriptivo, donde se realizó encuesta en línea a cirujanos recién egresados en 2015 y 2016, con preguntas de autoevaluación de competencias para resolver los traumas más comunes y más graves junto a ayudante pasivo. Se utilizó escala de Likert de 5 puntos.
Fueron evaluados 24 procedimientos de trauma cervical, torácico, abdominal y vascular. Se consultó el número de procedimientos asociados realizados durante su formación. Resultados: Se incluyeron 86 cirujanos recién egresados, de 11 programas de formación diferentes. La sensación de seguridad que presentaron es alta en procedimientos como lesiones intestinales y trauma vesical, donde el $100 \%$ y $77 \%$ se sienten competentes o muy competentes en su reparación. Sin embargo, en traumas complejos como el cervical hasta un $88 \%$ refiere sentirse con baja capacidad de resolución. Igualmente, en trauma vascular, la mayoría se siente incompetente por ejemplo para reparar una lesión de arteria axilar (92\%) y una ruptura aneurisma aórtico infrarenal (89\%). El 62\% realizó más de 10 laparotomías exploradoras, sin embargo, un $23 \%$ nunca realizó una toracotomía, 31\% ningún puente vascular, $49 \%$ ninguna sutura pulmonar, $84 \%$ ninguna sutura de aorta, $68 \%$ ninguna sutura cardíaca y $40 \%$ ninguna fístula arterio-venosa. Conclusiones: Los programas de cirugía actuales entregan herramientas útiles en la resolución de patologías frecuentes, sin embargo, en trauma existen falencias en cuanto al número de procedimientos realizados y bajas competencias en casos complejos.

Morbidity and Mortality Conferences: A Key Step Towards Quality Improvement Programs in Peru. Gabriela Zavala, Willy J Neumann, Eduardo HEgoavil, Manuel JAR Castro, N Charles, Lacey N Lagrone. From the Universidad Peruana Cayetano Heredia, Lima, Peru.

Introduction: IIInesses treatable with emergency surgery account for 116 million disability-adjusted life years lost globally each year. Trauma quality improvement programs (TQIP) have been shown to improve outcomes and decrease cost across resource settings. We present serial assessment data of TQIP in Peru to determine implementation trend of these important programs. Materials and methods: We conducted an anonymous written questionnaire which included respondent demographics, hospital descriptors, objective QI practices and subjective factors regarding morbidity and mortality (M\&M) conferences. We tested for significance using Chi-squared analysis. Results: 105 surgeons, medical students, residents, and nurses responded to a cross-sectional survey. Eighty four percent of respondents reported that M\&M's occur at their hospital compared to $97 \%$ reported in 2016 . Thirty five percent of conferences were reported as weekly in 2019, compared to $39 \%$ in $2016,23 \%$ occurred less than every three months in 2019 , compared to $27 \%$ in 2016 . In 2019, 60\% reported at least three staff physicians attended conferences, compared to $80 \%$ in 2016. In 2016 and 2019 surveys reported 50\% of some form of trauma registry in hospitals. Standardized case selection criteria, written documentation of M\&M conference proceedings and a plan for discussion follow-up were previously identified to be predictive of effective M\&M's. In 2016, 14\% of surveys reported standardized case selection criteria, 38\% included written documentation in M\&M's and 42\% follow-up plan after M\&M's; whereas in 2019, 17\% of questionnaires reported standardized case selection criteria, $37 \%$ included written documentation and $27 \%$ involved a follow-up plan. None of the differences reached statistical significance. Conclusions: Three years following an initial assessment of TQIP in Peru, we find similar practice patterns and areas for improvement. Given the strong evidence base for these interventions and the 
clearly identified areas for optimization of outcomes in Peruvian trauma patients, we recommend increased national-level prioritization of high quality M\&M conferences in Peru.

Simulación De Procedimientos Quirúrgicos De Trauma En Modelos Cadavericos: Experiencia. Javier Vela, Caterina Contreras, Juan P Ramos, Pablo Ottolino, Gabriel, Julian Varas, Pablo Achurra. From the Pontificia Universidad Católica De Chile, Santiago, Chile.

Introducción: La disminución en los horarios laborales de residentes junto a una baja exposición a trauma quirúrgico como primer cirujano, entre otros factores, no permiten completar la curva de aprendizaje de estas cirugías. La simulación en cadáveres es un ambiente seguro para el desarrollo de estas habilidades y podría complementar la formación. Objetivo: Evaluar las impresiones de cirujanos en formación de la enseñanza de procedimientos quirúrgicos mediante la simulación en modelos cadavéricos. Materiales y métodos: Estudio de corte transversal y descriptivo. De Abril 2018 a Abril 2019 residentes de cirugía general realizaron simulación quirúrgica cadavérica de procedimientos: esplenectomía, nefrectomías, colectomía, disecciones vasculares, masaje cardiaco directo, maniobras de Mattox, de Cathell Braasch, y de Kocher, entre otras. Se les aplicó una encuesta de percepción en línea con 5 preguntas tipo likert (1-5) interrogando las impresiones acerca del modelo de enseñanza, y 3 preguntas de respuesta abierta para ver aspectos a mejorar. Resultados: Respondieron la encuesta 22 personas, de los cuales $27.3 \%$ eran residentes de primer año de cirugía general, $31.8 \%$, de segundo año y $40.9 \%$ de tercer año. El $86 \%$ (19) está muy de acuerdo con que adquirió nuevo conocimiento. El $72.7 \%$ (16) está muy de acuerdo con que aprendió nuevas técnicas, $90.9 \%$ (20) está muy de acuerdo con que el cadáver ayuda a crear disecciones anatómicas más realistas. $63.6 \%$ (14) reporta estar muy de acuerdo con sentirse más seguro realizando este procedimiento en la práctica clínica. La mitad (11), está muy de acuerdo con que su nivel de confianza para realizar este procedimiento mejoró después del entrenamiento. Conclusiones: La simulación de procedimientos quirúrgicos de trauma en cadáveres es un procedimiento bien evaluado por los residentes de cirugía. Estos resultados son alentadores para evaluar la inclusión de estos procedimientos en la malla curricular de cirugía general.

Validación De Modelo Perfundido De Anastomosis Vascular Como Método De Evaluación De Competencias En Cirugía De Trauma. Javier Vela, Caterina Contreras, Cristián Jarry, Juan P Ramos, Pablo, Ignacio Torrealba, Julian Varas, Pablo Achurra. From the Pontificia Universidad Católica De Chile, Santiago, Chile.

Introducción: En Chile el trauma es la tercera causa de muerte en población general. Durante la formación de los cirujanos generales es difícil adquirir competencias en trauma vascular debido a la poca exposición a estos procedimientos, por lo que algunos no han realizado como primer cirujano una fístula arterio-venosa, sutura de aorta o puentes vasculares. Objetivo: Desarrollar un modelo simulado de anastomosis vascular en vasos grandes que permita evaluar las competencias de los cirujanos para realizar este procedimiento. Materiales y métodos: Se desarrolló un modelo ex vivo de anastomosis vascular usando arterias iliacas y aortas de porcino. Luego se evaluó a tres grupos de cirujanos (expertos, intermedios y novatos) realizando el procedimiento en el modelo. Se midió comparó el porcentaje de flujo arterial pre y post anastomosis, También se midió la duración del procedimiento. Resultados: Los grupos fueron compuestos por cirujanos expertos, intermedios y novatos. El flujo arterial tras la anastomosis fue superior al $90 \%$ del basal en los expertos, promedio $50 \%$ en los intermedios y menos de $30 \%$ del basal en los novatos. El tiempo operatorio también fue medido para cada grupo, siendo este menor en los expertos que no expertos, con una diferencia fue estadísticamente significativa. Conclusiones: El modelo presentado logra discriminar entre cirujanos expertos y no expertos en el procedimiento, por lo que es un modelo válido al momento de evaluar competencias quirúrgicas en anastomosis vascular en vasos grandes. Estos resultados permitirán a futuro desarrollar un programa de enseñanza para adquirir estas habilidades en cirujanos en formación.

Reparación De La Unión Gastroesofágica Transfixiante Posterior a Trauma Por Bala. Javier Vela, Caterina Contreras, Pablo Achurra, Marco Ceroni, Juan Pablo, Gabriel Escalona, Julian Varas, Alfonso Diaz. From the Pontificia Universidad Católica De Chile, Santiago, Chile.

Introducción: El traumatismo por bala de la unión gastroesofágica (UGE) es infrecuente, debido a la alta mortalidad de las lesiones vasculares asociadas, por lo que lo que no existe un estándar de tratamiento. Objetivo: Presentar un caso clínico y video de alta calidad (full HD), con la aproximación y técnica quirúrgica utilizada en un paciente con lesión transfixiante por bala de la UGE. Materiales y métodos: Se presenta un video de alta resolución con los datos clínicos, TAC y grabación de procedimiento qurúrgico de un paciente de 25 años evaluado en el servicio de urgencia con trauma de la región toracoabdominal por bala, con ingreso de proyectil por el tórax izquierdo. Resultados: Se realiza laparotomía, identificando trayecto de bala que atraviesa en forma lineal: (1) diafragma izquierdo; (2) segmento lateral del hígado; (3) cara anterior del fondo gástrico; (4) cara posterior de la UGE; (5) pilar diafragmático derecho, respetando grandes vasos. Se realiza sutura de la unión UGE a través de una gastrotomía anterior, fudoplicatura e intalación de drenaje hacia cara posterior de esófago y yeyunostomía. Conclusiones: El paciente intervenido evoluciona con fístula biliar de la lesión del segmento lateral que se manejó de forma conservadora, con el posterior retiro del drenaje al día 14 post operatorio. 